\title{
BILINEAR PSEUDO-DIFFERENTIAL OPERATORS WITH GEVREY-HÖRMANDER SYMBOLS
}

\author{
AHMED ABDELJAWAD, SANDRO CORIASCO, AND NENAD TEOFANOV
}

\begin{abstract}
We consider bilinear pseudo-differential operators whose symbols posses Gevrey type regularity and may have a sub-exponential growth at infinity, together with all their derivatives. It is proved that those symbol classes can be described by the means of the short-time Fourier transform and modulation spaces. Our first main result is the invariance property of the corresponding bilinear operators. Furthermore we prove the continuity of such operators when acting on modulation spaces. As a consequence, we derive their continuity on anisotropic Gelfand-Shilov type spaces. We consider both Beurling and Roumieu type symbol classes and Gelfand-Shilov spaces.
\end{abstract}

\section{INTRODUCTION}

The study of multilinear operators has been influenced by the Calderón-Zygmund theory. Indeed, one of the achievements of CoifmanMeyer's pioneer work [11] is the realization of pseudo-differential operators in terms of singular integrals of Calderón-Zygmund type. Their approach is based on a multilinear point of view and have had a far reaching impact in operator theory and partial differential equations. For example, boundedness of a class of translation invariant bilinear operators on Lebesgue spaces is proved in [11]. Furthermore, the bilinear Calderón-Zygmund theory developed by Grafakos and Torres [22] paved the way to the extension of those results to bilinear pseudodifferential operators which are non-translation invariant, i.e. whose symbols may depend on the space variable as well. We refer to [4] for a brief survey and discussion of applications to partial differential equations, and to [8] for a systematic study of bilinear pseudo-differential operators with symbols in bilinear Hörmander classes. See also [28] for a recent contribution in the context of Triebel-Lizorkin and local Hardy spaces.

Another type of results concerns bilinear (and multilinear) operators whose symbols are not necessarily smooth. Their continuity properties on modulation spaces were first observed in [5]. In contrast to classical bilinear pseudo-differential operators considered in e.g. [11], these

2010 Mathematics Subject Classification. 35S05, 47B37, 47G30, 42B35.

Key words and phrases. Bilinear operator, Pseudo-differential operators, Modulation spaces, Gelfand-Shilov spaces, Gevrey regularity. 
operators are treated by the techniques of time-frequency analysis, see also [6 8, 13, 31].

In this paper, we employ the techniques of time-frequency analysis and modulation spaces, and consider bilinear pseudo-differential operators of Gevrey-Hörmander type whose symbols are of infinite order and may have a (super-)exponential growth at infinity, together with all their derivatives. The linear counterpart of such operators is considered in [9], within the environment of isotropic Gelfand-Shilov spaces of functions and distributions, see also [2, 49], and extended to the anisotropic setting in [1, 3]. The main purpose of this paper is to extend some boundedness results given there to the bilinear case.

More precisely, we consider Gevrey-Hörmander type symbols $a \in$ $\Gamma_{(\omega)}^{\sigma, \mathbf{s}}\left(\mathbf{R}^{3 d}\right)$ (or $a \in \Gamma_{(\omega)}^{\sigma, \mathbf{s}, 0}\left(\mathbf{R}^{3 d}\right)$ ), see Definition 1.9, and the corresponding pseudo-differential operators, denoted by $\mathrm{Op}_{r, t}(a)$, see (1.14) below. When $r=t=0$ we recover the Kohn-Nirenberg correspondence considered, e.g., in [5, 7], while for $r=t=1 / 2$ we obtain the Weyl correspondence, considered in [39,41]. We remark that, in view of this choice of symbol classes, we cannot rely on arguments based on standard (e.g., Littlewood-Paley) localization techniques. The substitute for this is, from the very beginning, a "global approach", aimed at obtaining and employing appropriate characterizations of the involved objects, in terms of suitable estimates which hold true on the whole Euclidean spaces.

The paper is organized as follows. In Section 1 we collect necessary definitions, background material and basic facts on Gelfand-Shilov spaces, weight functions, modulation spaces, symbol classes and the corresponding bilinear pseudo-differential operators. In Section 2, we first study exponential-type operators on Gelfand-Shilov space and prove the corresponding invariance properties. We proceed with a characterization of the symbol spaces in terms of their regularity and decay properties, and suitable estimates related to modulation spaces. Finally, we prove our main results about the continuity of bilinear operators in Section 3. The proofs of a few technical results are collected in the Appendix.

\section{Preliminaries}

In this section we provide notation and background material which will be used throughout the paper. Proofs and details are in general omitted, since they can be found, e.g., in [12, 14 19, 24, 39, 42, 44].

We use the standard notation for Euclidean spaces and multiindeces, cf. 27]. For example, if $x=\left(x_{1}, \ldots, x_{d}\right) \in \mathbf{R}^{d}$ and $\alpha=\left(\alpha_{1}, \ldots, \alpha_{d}\right) \in \mathbf{N}^{d}$, then $x^{\alpha}=x_{1}^{\alpha_{1}} \ldots x_{d}^{\alpha_{d}}, \partial_{x}^{\alpha}=\partial_{x_{1}}^{\alpha_{1}} \ldots \partial_{x_{d}}^{\alpha_{d}},|\alpha|=\alpha_{1}+\cdots+\alpha_{d}$ and $\alpha$ ! $=$ $\alpha_{1}$ ! ... $\alpha_{d}$ !. Here $\mathbf{N}$ denotes the set of non-negative integers. If $\alpha \in \mathbf{N}^{d}$, then $\alpha>0$ means that $\alpha_{j}>0$ for every $j=1, \ldots, d$, and similarly 
for $\alpha \geq 0$. We write $A(\theta) \lesssim B(\theta), \theta \in \Omega$, if there is a constant $c>0$ such that $|A(\theta)| \leq c|B(\theta)|$ for all $\theta \in \Omega$. We write $A(\theta) \asymp B(\theta)$ if $A(\theta) \lesssim B(\theta)$ and $B(\theta) \lesssim A(\theta)$ for all $\theta \in \Omega$. Here $\Omega$ is an open subset of $\mathbf{R}^{N}$. If $\mathcal{B}_{1}$ and $\mathcal{B}_{2}$ are topological vector spaces, then $\mathcal{B}_{1} \hookrightarrow \mathcal{B}_{2}$ means that $\mathcal{B}_{1}$ is continuously embedded into $\mathcal{B}_{2}$. By $\mathscr{S}\left(\mathbf{R}^{d}\right)$ we denote the Schwartz space of rapidly decreasing functions, and $\mathscr{S}^{\prime}\left(\mathbf{R}^{d}\right)$ denotes its dual space of tempered distributions.

1.1. Gelfand-Shilov spaces. Let $h, s, \sigma>0$ be fixed. Then $\mathcal{S}_{s ; h}^{\sigma}\left(\mathbf{R}^{d}\right)$ is the Banach space of all $f \in C^{\infty}\left(\mathbf{R}^{d}\right)$ such that

$$
\|f\|_{\mathcal{S}_{s ; h}^{\sigma}} \equiv \sup _{\alpha, \beta \in \mathbf{N}^{d}} \sup _{x \in \mathbf{R}^{d}} \frac{\left|x^{\alpha} \partial^{\beta} f(x)\right|}{h^{|\alpha+\beta|} \alpha !^{\prime} \beta ! \sigma}<\infty,
$$

endowed with the norm (1.1). Obviously, $\mathcal{S}_{s ; h}^{\sigma}\left(\mathbf{R}^{d}\right)$ increases as $h, s$ and $\sigma$ increase, and it is contained in $\mathscr{S}\left(\mathbf{R}^{d}\right)$ for every $h, s, \sigma>0$.

The Gelfand-Shilov spaces $\mathcal{S}_{s}^{\sigma}\left(\mathbf{R}^{d}\right)$ and $\Sigma_{s}^{\sigma}\left(\mathbf{R}^{d}\right)$ are defined as the inductive and projective limits respectively of $\mathcal{S}_{s ; h}^{\sigma}\left(\mathbf{R}^{d}\right)$, i.e.

$$
\mathcal{S}_{s}^{\sigma}\left(\mathbf{R}^{d}\right)=\bigcup_{h>0} \mathcal{S}_{s ; h}^{\sigma}\left(\mathbf{R}^{d}\right) \quad \text { and } \quad \Sigma_{s}^{\sigma}\left(\mathbf{R}^{d}\right)=\bigcap_{h>0} \mathcal{S}_{s ; h}^{\sigma}\left(\mathbf{R}^{d}\right),
$$

with the usual inductive and projective limit topologies. Note that $\Sigma_{s}^{\sigma}\left(\mathbf{R}^{d}\right) \neq\{0\}$, if and only if $s+\sigma \geq 1$ and $(s, \sigma) \neq\left(\frac{1}{2}, \frac{1}{2}\right)$, and $\mathcal{S}_{s}^{\sigma}\left(\mathbf{R}^{d}\right) \neq\{0\}$, if and only if $s+\sigma \geq 1$, see [21,32]. For every $s, \sigma>0$ we have

$$
\Sigma_{s}^{\sigma}\left(\mathbf{R}^{d}\right) \hookrightarrow \mathcal{S}_{s}^{\sigma}\left(\mathbf{R}^{d}\right) \hookrightarrow \Sigma_{s+\varepsilon}^{\sigma+\varepsilon}\left(\mathbf{R}^{d}\right) \hookrightarrow \mathscr{S}\left(\mathbf{R}^{d}\right)
$$

for every $\varepsilon>0$. If $s+\sigma \geq 1$, then the last two inclusions in (1.3) are dense, and if in addition $(s, \sigma) \neq\left(\frac{1}{2}, \frac{1}{2}\right)$ then the first inclusion in (1.3) is dense. Moreover, for $\sigma<1$ the elements of $\mathcal{S}_{s}^{\sigma}\left(\mathbf{R}^{d}\right)$ admit entire extensions to $\mathbf{C}^{d}$ satisfying suitable exponential bounds, [21].

Remark 1.1. Note that the original definition in [21] is given with $s, \sigma \geq 0$. Then $\mathcal{S}_{0}^{\sigma}\left(\mathbf{R}^{d}\right) \neq\{0\}\left(\Sigma_{0}^{\sigma}\left(\mathbf{R}^{d}\right) \neq\{0\}\right)$ and $\mathcal{S}_{s}^{0}\left(\mathbf{R}^{d}\right) \neq\{0\}$ $\left(\Sigma_{s}^{0}\left(\mathbf{R}^{d}\right) \neq\{0\}\right)$, if and only if $\sigma>1$ and $s>1$ respectively, see [21, Chapter IV 8.1]. In fact, $\mathcal{S}_{0}^{\sigma}\left(\mathbf{R}^{d}\right) \neq\{0\}$ consists of compactly supported Gevrey functions, while $\mathcal{S}_{s}^{0}\left(\mathbf{R}^{d}\right) \neq\{0\}$ contains functions whose Fourier transforms are compactly supported Gevrey functions.

The spaces $\mathcal{S}_{s}^{\sigma}\left(\mathbf{R}^{d}\right)$ and $\Sigma_{s}^{\sigma}\left(\mathbf{R}^{d}\right)$ combine global regularity with suitable decay properties at infinity, thus offering an abstract functional analytic framework for some problems in mathematical physics, [23, 30]. The following result is a well-known characterization of $\mathcal{S}_{s}^{\sigma}\left(\mathbf{R}^{d}\right)$ and $\Sigma_{s}^{\sigma}\left(\mathbf{R}^{d}\right)$ in terms of the exponential decay of derivatives of their elements. Although the proof is standard, it contains some tools relevant for our investigations, and is therefore included in Appendix A. 
Lemma 1.2. Let $f$ be a smooth function on $\mathbf{R}^{d}, f \in C^{\infty}\left(\mathbf{R}^{d}\right)$. Then $f \in \mathcal{S}_{s}^{\sigma}\left(\mathbf{R}^{d}\right)$ (respectively $f \in \Sigma_{s}^{\sigma}\left(\mathbf{R}^{d}\right)$ ) if and only if for every $\alpha \in \mathbf{N}^{d}$

$$
\left|\partial^{\alpha} f(x)\right| \lesssim l^{|\alpha|} \alpha !^{\sigma} e^{-h|x|^{\frac{1}{s}}}, \quad x \in \mathbf{R}^{d},
$$

for some $l, h>0$ (respectively for every $l, h>0$ ).

The Gelfand-Shilov distribution spaces $\left(\mathcal{S}_{s}^{\sigma}\right)^{\prime}\left(\mathbf{R}^{d}\right)$ and $\left(\Sigma_{s}^{\sigma}\right)^{\prime}\left(\mathbf{R}^{d}\right)$ are the projective and inductive limit respectively of $\left(\mathcal{S}_{s ; h}^{\sigma}\right)^{\prime}\left(\mathbf{R}^{d}\right)$ :

$$
\left(\mathcal{S}_{s}^{\sigma}\right)^{\prime}\left(\mathbf{R}^{d}\right)=\bigcap_{h>0}\left(\mathcal{S}_{s ; h}^{\sigma}\right)^{\prime}\left(\mathbf{R}^{d}\right) \quad \text { and } \quad\left(\Sigma_{s}^{\sigma}\right)^{\prime}\left(\mathbf{R}^{d}\right)=\bigcup_{h>0}\left(\mathcal{S}_{s ; h}^{\sigma}\right)^{\prime}\left(\mathbf{R}^{d}\right) .
$$

It follows that $\mathscr{S}^{\prime}\left(\mathbf{R}^{d}\right) \hookrightarrow\left(\mathcal{S}_{s}^{\sigma}\right)^{\prime}\left(\mathbf{R}^{d}\right)$ when $s+\sigma \geq 1$, and if in addition $(s, \sigma) \neq\left(\frac{1}{2}, \frac{1}{2}\right)$, then $\left(\mathcal{S}_{s}^{\sigma}\right)^{\prime}\left(\mathbf{R}^{d}\right) \hookrightarrow\left(\Sigma_{s}^{\sigma}\right)^{\prime}\left(\mathbf{R}^{d}\right)$.

The Fourier transform $\mathscr{F}$ is the linear and continuous map on $\mathscr{S}\left(\mathbf{R}^{d}\right)$, given by the formula

$$
(\mathscr{F} f)(\xi)=\widehat{f}(\xi) \equiv(2 \pi)^{-\frac{d}{2}} \int_{\mathbf{R}^{d}} f(x) e^{-i\langle x, \xi\rangle} d x, \quad \xi \in \mathbf{R}^{d},
$$

when $f \in \mathscr{S}\left(\mathbf{R}^{d}\right)$. Here $\langle\cdot, \cdot\rangle$ denotes the usual scalar product on $\mathbf{R}^{d}$. The Fourier transform extends uniquely to homeomorphisms from $\left(\mathcal{S}_{s}^{\sigma}\right)^{\prime}\left(\mathbf{R}^{d}\right)$ to $\left(\mathcal{S}_{\sigma}^{s}\right)^{\prime}\left(\mathbf{R}^{d}\right)$, and from $\left(\Sigma_{s}^{\sigma}\right)^{\prime}\left(\mathbf{R}^{d}\right)$ to $\left(\Sigma_{\sigma}^{s}\right)^{\prime}\left(\mathbf{R}^{d}\right)$. Furthermore, it restricts to homeomorphisms from $\mathcal{S}_{s}^{\sigma}\left(\mathbf{R}^{d}\right)$ to $\mathcal{S}_{\sigma}^{s}\left(\mathbf{R}^{d}\right)$, and from $\Sigma_{s}^{\sigma}\left(\mathbf{R}^{d}\right)$ to $\Sigma_{\sigma}^{s}\left(\mathbf{R}^{d}\right)$.

Next we rewrite the definition of Gelfand-Shilov spaces in the notation which is convenient for our analysis, see also [9, 21, 26]. We put

$$
\mathbf{R}^{d_{0}+\cdots+d_{k}}=\mathbf{R}^{d_{0}} \times \mathbf{R}^{d_{1}} \times \cdots \times \mathbf{R}^{d_{k}}=\mathbf{R}^{\left(d_{0}, \ldots, d_{k}\right)}=\mathbf{R}^{\mathbf{d}} .
$$

Definition 1.3. Let $k \in \mathbf{N}, \sigma=\left(\sigma_{0}, \ldots, \sigma_{k}\right)>0, \mathbf{s}=\left(s_{0}, \ldots, s_{k}\right)>0$, and $\mathbf{d}=d_{0}+\cdots+d_{k}$. The Gelfand-Shilov spaces

$$
\mathcal{S}_{\mathbf{s}}^{\sigma}\left(\mathbf{R}^{\mathbf{d}}\right)=\mathcal{S}_{s_{0}, \ldots, s_{k}}^{\sigma_{0}, \ldots, \sigma_{k}}\left(\mathbf{R}^{d_{0}+\cdots+d_{k}}\right) \quad \text { and } \quad \Sigma_{\mathbf{s}}^{\sigma}\left(\mathbf{R}^{\mathbf{d}}\right)=\Sigma_{s_{0}, \ldots, s_{k}}^{\sigma_{0}, \ldots, \sigma_{k}}\left(\mathbf{R}^{d_{0}+\cdots+d_{k}}\right),
$$

consist of all $F \in C^{\infty}\left(\mathbf{R}^{d_{0}+\cdots+d_{k}}\right)$ such that

$$
\left|x_{0}^{\alpha_{0}} \ldots x_{k}^{\alpha_{k}} \partial_{x_{0}}^{\beta_{0}} \ldots \partial_{x_{k}}^{\beta_{k}} F\left(x_{0}, \ldots, x_{k}\right)\right| \lesssim h^{\left|\alpha_{0}+\beta_{0}+\cdots+\alpha_{k}+\beta_{k}\right|} \prod_{j=0}^{k} \alpha_{j} !^{s_{j}} \beta_{j} !^{\sigma_{j}}
$$

for some $h>0$ and for every $h>0$ respectively, where $x_{j} \in \mathbf{R}^{d_{j}}$, $\alpha_{j}, \beta_{j} \in \mathbf{N}^{d_{j}}, j=0, \ldots, k$. The dual spaces of $\mathcal{S}_{\mathbf{S}}^{\sigma}\left(\mathbf{R}^{\mathbf{d}}\right)$ and $\Sigma_{\mathbf{s}}^{\sigma}\left(\mathbf{R}^{\mathbf{d}}\right)$ are denoted by

$$
\left(\mathcal{S}_{\mathbf{s}}^{\sigma}\right)^{\prime}\left(\mathbf{R}^{\mathbf{d}}\right)=\left(\mathcal{S}_{s_{0}, \ldots, s_{k}}^{\sigma_{0}, \ldots, \sigma_{k}}\right)^{\prime}\left(\mathbf{R}^{d_{0}+\cdots+d_{k}}\right)
$$

and

$$
\left(\sum_{s_{0}, \ldots, s_{k}}^{\sigma_{0}, \ldots, \sigma_{k}}\right)^{\prime}\left(\mathbf{R}^{\mathbf{d}}\right)=\left(\Sigma_{s_{0}, \ldots, s_{k}}^{\sigma_{0}, \ldots, \sigma_{k}}\right)^{\prime}\left(\mathbf{R}^{d_{0}+\cdots+d_{k}}\right)
$$

respectively. 
The space $\mathcal{S}_{\mathbf{s}}^{\sigma}\left(\mathbf{R}^{\mathbf{d}}\right)$ is nontrivial if and only if $s_{j}+\sigma_{j} \geq 1$, for each $j=0, \ldots, k$ and $\Sigma_{\mathbf{s}}^{\sigma}\left(\mathbf{R}^{\mathbf{d}}\right)$ is nontrivial if and only if $s_{j}+\sigma_{j} \geq 1$, and $\left(s_{j}, \sigma_{j}\right) \neq\left(\frac{1}{2}, \frac{1}{2}\right)$ for each $j=0, \ldots, k$.

Obviously, if $\sigma_{j}=\sigma, s_{j}=s$ and $d_{j}=d, j=0, \ldots, k$, then

$$
\begin{gathered}
\mathcal{S}_{s_{0}, \ldots, s_{k}}^{\sigma_{0}, \ldots, \sigma_{k}}\left(\mathbf{R}^{d_{0}+\cdots+d_{k}}\right) \equiv \mathcal{S}_{s}^{\sigma}\left(\mathbf{R}^{(k+1) d}\right), \quad \sum_{s_{0}, \ldots, s_{k}}^{\sigma_{0}, \ldots, \sigma_{k}}\left(\mathbf{R}^{d_{0}+\cdots+d_{k}}\right) \equiv \Sigma_{s}^{\sigma}\left(\mathbf{R}^{(k+1) d}\right) \\
\left(\mathcal{S}_{s_{0}, \ldots, s_{k}}^{\sigma_{0}, \ldots, \sigma_{k}}\right)^{\prime}\left(\mathbf{R}^{d_{0}+\cdots+d_{k}}\right) \equiv\left(\mathcal{S}_{s}^{\sigma}\right)^{\prime}\left(\mathbf{R}^{(k+1) d}\right)
\end{gathered}
$$

and

$$
\left(\Sigma_{s_{0}, \ldots, s_{k}}^{\sigma_{0}, \ldots, \sigma_{k}}\right)^{\prime}\left(\mathbf{R}^{d_{0}+\cdots+d_{k}}\right) \equiv\left(\Sigma_{s}^{\sigma}\right)^{\prime}\left(\mathbf{R}^{(k+1) d}\right) .
$$

The Fourier transform is a homeomorphism between $\mathcal{S}_{s}^{\sigma}\left(\mathbf{R}^{d}\right)$ and $\mathcal{S}_{\sigma}^{s}\left(\mathbf{R}^{d}\right)$ (and between $\Sigma_{s}^{\sigma}\left(\mathbf{R}^{d}\right)$ and $\Sigma_{\sigma}^{s}\left(\mathbf{R}^{d}\right)$ ), cf. [21]. This, together with the kernel theorem for Gelfand-Shilov spaces (see [29, 34, 38] implies the following mapping properties of partial Fourier transforms on GelfandShilov spaces. The proof is therefore omitted. Here, $\mathscr{F}_{j} F$ is the partial Fourier transforms of $F\left(x_{0}, x_{1}, \ldots, x_{k}\right)$ with respect to $x_{j} \in \mathbf{R}^{d_{j}}, j=$ $0, \ldots, k$.

Proposition 1.4. Let $k \in \mathbf{N}, s_{j}, \sigma_{j}>0, j=0, \ldots, k$. Then the following is true:

(1) the mapping $\mathscr{F}_{j}$ on $\mathscr{S}\left(\mathbf{R}^{d_{0}+\cdots+d_{k}}\right)$ restrict to homeomorphism

$$
\mathscr{F}_{j}: \mathcal{S}_{s_{0}, \ldots, s_{k}}^{\sigma_{0}, \ldots, \sigma_{k}}\left(\mathbf{R}^{d_{0}+\cdots+d_{k}}\right) \rightarrow \mathcal{S}_{s_{0}, \ldots, s_{j-1}, \sigma_{j}, s_{j+1}, \ldots, s_{k}}^{\sigma_{0}, \ldots, \sigma_{j-1}, s_{j}, \sigma_{j+1}, \ldots, \sigma_{k}}\left(\mathbf{R}^{d_{0}+\cdots+d_{k}}\right) ;
$$

(2) the mapping $\mathscr{F}_{j}$ on $\mathscr{S}\left(\mathbf{R}^{d_{0}+\cdots+d_{k}}\right)$ is uniquely extendable to homeomorphism

$$
\mathscr{F}_{j}:\left(\mathcal{S}_{s_{0}, \ldots, s_{k}}^{\sigma_{0}, \ldots, \sigma_{k}}\right)^{\prime}\left(\mathbf{R}^{d_{0}+\cdots+d_{k}}\right) \rightarrow\left(\mathcal{S}_{s_{0}, \ldots, s_{j-1}, \sigma_{j}, s_{j+1}, \ldots, s_{k}}^{\sigma_{0}, \ldots, \sigma_{j-1}, s_{j}, \sigma_{j+1}, \ldots, \sigma_{k}}\right)^{\prime}\left(\mathbf{R}^{d_{0}+\cdots+d_{k}}\right) .
$$

The same holds true if the $\mathcal{S}_{s_{0}, \ldots, s_{k}}^{\sigma_{0}, \ldots, \sigma_{k}}$-spaces and their duals are replaced by corresponding $\sum_{s_{0}, \ldots, s_{k}}^{\sigma_{0}, \ldots, \sigma_{k}}$-spaces and their duals in each occurrence.

The result analogous to Proposition 1.4 holds for partial Fourier transforms with respect to some choice of variables. In particular the (full) Fourier transform on $\mathscr{S}\left(\mathbf{R}^{\mathbf{d}}\right)$ restricts to to homeomorphism

$$
\mathscr{F}: \mathcal{S}_{\mathbf{s}}^{\sigma}\left(\mathbf{R}^{\mathbf{d}}\right) \rightarrow \mathcal{S}_{\sigma}^{\mathbf{s}}\left(\mathbf{R}^{\mathbf{d}}\right)
$$

and is uniquely extendable to homeomorphism

$$
\mathscr{F}:\left(\mathcal{S}_{\mathbf{s}}^{\sigma}\right)^{\prime}\left(\mathbf{R}^{\mathbf{d}}\right) \rightarrow\left(\mathcal{S}_{\sigma}^{\mathbf{s}}\right)^{\prime}\left(\mathbf{R}^{\mathbf{d}}\right)
$$

and same holds true if $\mathcal{S}_{\mathbf{s}}^{\sigma}\left(\mathbf{R}^{\mathbf{d}}\right)$ spaces and their duals are replaced by corresponding $\Sigma_{\mathbf{s}}^{\sigma}\left(\mathbf{R}^{\mathbf{d}}\right)$ spaces and their duals.

Alternatively, this result is contained in the following Proposition.

Proposition 1.5. Let $k \in \mathbf{N}, \sigma=\left(\sigma_{0}, \ldots, \sigma_{k}\right)>0, \mathbf{s}=\left(s_{0}, \ldots, s_{k}\right)>$ 0 , and $\mathbf{d}=d_{1}+\cdots+d_{k}$. Then the following conditions are equivalent.

(1) $F \in \mathcal{S}_{\mathbf{s}}^{\sigma}\left(\mathbf{R}^{\mathbf{d}}\right) \quad\left(F \in \Sigma_{\mathbf{s}}^{\sigma}\left(\mathbf{R}^{\mathbf{d}}\right)\right)$; 
(2) for some $r>0$ (for every $r>0$ ) it holds

$$
\left|F\left(x_{0}, \ldots, x_{k}\right)\right| \lesssim e^{-r\left(\left|x_{0}\right|^{\frac{1}{s_{0}}}+\cdots+\left|x_{k}\right|^{\frac{1}{s_{k}}}\right)}
$$

and

$$
\left|\widehat{F}\left(\xi_{0}, \ldots, \xi_{k}\right)\right| \lesssim e^{-r\left(\left|\xi_{0}\right|^{\frac{1}{\sigma_{0}}}+\cdots+\left|\xi_{k}\right|^{\frac{1}{\sigma_{k}}}\right)}
$$

(3) for every $\alpha=\left(\alpha_{0}, \ldots, \alpha_{k}\right) \in \mathbf{N}^{\mathbf{d}}$ and for some $h, r>0$ (for every $h, r>0)$ it holds

$$
\left|\partial^{\alpha} F\left(x_{0}, \ldots, x_{k}\right)\right| \lesssim h^{|\alpha|} \prod_{j=0}^{k} \alpha_{j}^{\sigma_{j}} e^{-r\left(\left|x_{0}\right|^{\frac{1}{s_{0}}}+\cdots+\left|x_{k}\right|^{\frac{1}{s_{k}}}\right)} .
$$

Proof. The equivalence between (1) and (2) follows from [10], and (1) $\Leftrightarrow(2)$ can be proved by a slight modification of the proof of Lemma 1.2 (cf. Appendix A) and we therefore leave it for the reader.

1.2. Weight functions. A function $\omega$ is called a weight or weight function on $\mathbf{R}^{d}$, if $\omega, 1 / \omega \in L_{l o c}^{\infty}\left(\mathbf{R}^{d}\right)$ are positive everywhere. Without loss of generality we may assume that the weight functions are continuous on $\mathbf{R}^{d}$. Let $\omega$ and $v$ be weights on $\mathbf{R}^{d}$. Then $\omega$ is called $v$-moderate or moderate, if

$$
\omega\left(x_{1}+x_{2}\right) \lesssim \omega\left(x_{1}\right) v\left(x_{2}\right), \quad x_{1}, x_{2} \in \mathbf{R}^{d} .
$$

If $v$ can be chosen as polynomial, then $\omega$ is called a weight of polynomial type. A weight function $v$ is submultiplicative, if it is symmetric in each coordinate and

$$
v\left(x_{1}+x_{2}\right) \lesssim v\left(x_{1}\right) v\left(x_{2}\right), \quad x_{1}, x_{2} \in \mathbf{R}^{d} .
$$

From now on, $v$ always denote a submultiplicative weight if nothing else is stated. In particular, if (1.5) holds and $v$ is submultiplicative, then

$$
\frac{\omega\left(x_{1}\right)}{v\left(x_{2}\right)} \lesssim \omega\left(x_{1}+x_{2}\right) \lesssim \omega\left(x_{1}\right) v\left(x_{2}\right), \quad x_{1}, x_{2} \in \mathbf{R}^{d} .
$$

If $\omega$ is a moderate weight on $\mathbf{R}^{d}$, then there exists a submultiplicative weight $v$ on $\mathbf{R}^{d}$ such that (1.5) and (1.6) hold, cf. [44, 45, 47]. Moreover if $v$ is submultiplicative on $\mathbf{R}^{d}$, then

$$
1 \lesssim v(x) \lesssim e^{c|x|}
$$

for some constant $c>0$ (cf. [25, Lemma 4.2]).

In particular, if $\omega$ is moderate, then

$$
\omega(x+y) \lesssim \omega(x) e^{c|y|} \quad \text { and } \quad e^{-c|x|} \lesssim \omega(x) \lesssim e^{c|x|}, \quad x, y \in \mathbf{R}^{d}
$$

for some $c>0$. 
For a given $k \in \mathbf{N}$, we let $\mathscr{P}_{E}\left(\mathbf{R}^{d_{0}+\cdots+d_{k}}\right)$ be the set of all moderate weights on $\mathbf{R}^{d_{0}+\cdots+d_{k}}$, and $\mathscr{P}\left(\mathbf{R}^{d_{0}+\cdots+d_{k}}\right)$ be the subset of $\mathscr{P}_{E}\left(\mathbf{R}^{d_{0}+\cdots+d_{k}}\right)$ which consists of weights of polynomial type.

If $\omega \in \mathscr{P}_{E}\left(\mathbf{R}^{d_{0}+\cdots+d_{k}}\right)$ then there exists a submultiplicative weight $v$ on $\mathbf{R}^{d_{0}+\cdots+d_{k}}$, such that

$$
\frac{\omega\left(x_{0}, \ldots, x_{k}\right)}{v\left(y_{0}, \ldots, y_{k}\right)} \lesssim \omega\left(x_{0}+y_{0}, \ldots, x_{k}+y_{k}\right) \lesssim \omega\left(x_{0}, \ldots, x_{k}\right) v\left(y_{0}, \ldots, y_{k}\right),
$$

where $x_{j}, y_{j} \in \mathbf{R}^{d_{j}}, j=0, \ldots, k$. Note that (1.7) for a submultiplicative weight $v$ on $\mathbf{R}^{d_{0}+\cdots+d_{k}}$ becomes

$$
1 \lesssim v\left(x_{0}, \ldots, x_{k}\right) \lesssim e^{r\left(\left|x_{0}\right|+\cdots+\left|x_{k}\right|\right)}, \quad x_{j} \in \mathbf{R}^{d_{j}}, j=0, \ldots, k,
$$

for some $r>0$.

Next we extend the weight functions considered in [1,2] to the case when $\mathbf{R}^{d}=\mathbf{R}^{d_{0}+\cdots+d_{k}}$.

Definition 1.6. Let $k \in \mathbf{N}$ and $s_{j}>0, j=0, \ldots, k$. Then, the set $\mathscr{P}_{s_{0}, \ldots, s_{k}}\left(\mathbf{R}^{d_{0}+\cdots+d_{k}}\right)\left(\mathscr{P}_{s_{0}, \ldots, s_{k}}^{0}\left(\mathbf{R}^{d_{0}+\cdots+d_{k}}\right)\right)$ consists of all weights $\omega \in$ $\mathscr{P}_{E}\left(\mathbf{R}^{d_{0}+\cdots+d_{k}}\right)$ such that

$$
\omega\left(x_{0}+y_{0}, \ldots, x_{k}+y_{k}\right) \lesssim \omega\left(x_{0}, \ldots, x_{k}\right) e^{r\left(\left|y_{0}\right|^{\frac{1}{s_{0}}}+\cdots+\left|y_{k}\right|^{\frac{1}{s_{k}}}\right)}, x_{j}, y_{j} \in \mathbf{R}^{d_{j}}
$$

holds for some (for every) $r>0$.

In particular, if $\omega \in \mathscr{P}_{s_{0}, \ldots, s_{k}}\left(\mathbf{R}^{d_{0}+\cdots+d_{k}}\right)\left(\mathscr{P}_{s_{0}, \ldots, s_{k}}^{0}\left(\mathbf{R}^{d_{0}+\cdots+d_{k}}\right)\right)$, then

$$
e^{-r\left(\left|x_{0}\right|^{\frac{1}{s_{0}}}+\cdots+\left|x_{k}\right|^{\frac{1}{s_{k}}}\right)} \lesssim \omega\left(x_{0}, \ldots, x_{k}\right) \lesssim e^{r\left(\left|x_{0}\right|^{\frac{1}{s_{0}}}+\cdots+\left|x_{k}\right|^{\frac{1}{s_{k}}}\right)}
$$

for some $r>0$ (for every $r>0$ ).

By (1.8) and (1.9) it follows that

$$
\mathscr{P}_{s_{0}, \ldots, s_{k}}^{0}\left(\mathbf{R}^{d_{0}+\cdots+d_{k}}\right)=\mathscr{P}_{\tilde{s}_{0}, \ldots, \tilde{s}_{k}}\left(\mathbf{R}^{d_{0}+\cdots+d_{k}}\right)=\mathscr{P}_{E}\left(\mathbf{R}^{d_{0}+\cdots+d_{k}}\right)
$$

when $s_{j}<1$ and $\tilde{s}_{j} \leq 1, j=0, \ldots, k$. For convenience we set

$$
\mathscr{P}_{E}^{0}\left(\mathbf{R}^{d_{0}+\cdots+d_{k}}\right)=\mathscr{P}_{E, 1}^{0}\left(\mathbf{R}^{d_{0}+\cdots+d_{k}}\right) .
$$

The following extension of [2, Proposition 1.6], shows that for any weight in $\mathscr{P}_{E}\left(\mathbf{R}^{d_{0}+\cdots+d_{k}}\right)$, there are equivalent weights that satisfy the anisotropic Gevrey regularity.

Proposition 1.7. Let there be given $\omega \in \mathscr{P}_{E}\left(\mathbf{R}^{d_{0}+d_{1}+\cdots+d_{k}}\right)$. Then there exists a weight $\omega_{0} \in \mathscr{P}_{E}\left(\mathbf{R}^{d_{0}+d_{1}+\cdots+d_{k}}\right) \cap C^{\infty}\left(\mathbf{R}^{d_{0}+d_{1}+\cdots+d_{k}}\right)$ such that the following is true:

(1) $\omega_{0} \asymp \omega$; 
(2) for every (multiindex) $\alpha_{j} \geq 0, j=0, \ldots, k$, we have

$$
\begin{gathered}
\left|\partial_{x}^{\alpha_{0}} \partial_{\xi_{1}}^{\alpha_{1}} \ldots \partial_{\xi_{k}}^{\alpha_{k}} \omega_{0}\left(x, \xi_{1}, \ldots, \xi_{k}\right)\right| \lesssim h^{\left|\alpha_{0}+\alpha_{1}+\cdots+\alpha_{k}\right|} \prod_{j=0}^{k} \alpha_{j} !^{s_{j}} \omega\left(x, \xi_{1}, \ldots, \xi_{k}\right) \\
\asymp h^{\left|\alpha_{0}+\alpha_{1}+\cdots+\alpha_{k}\right|} \prod_{j=0}^{k} \alpha_{j} !^{s_{j}} \omega_{0}\left(x, \xi_{1}, \ldots, \xi_{k}\right), \quad x \in \mathbf{R}_{0}^{d}, \xi_{j} \in \mathbf{R}^{d_{j}}, j=1, \ldots, k,
\end{gathered}
$$

for every $h>0$ and $s_{j}>0, j=0, \ldots, k$.

The proof is given in Appendix A.

1.3. Modulation spaces. Modulation spaces, originally introduced by Feichtinger in [16], are recognized as appropriate family of spaces when dealing with problems of time-frequency analysis, see [16 20, 24, 35, 37], to mention just a few references. A broader family of modulation spaces is recently studied in [2, 33, 49].

Let $s, \sigma>0$, such that $s+\sigma \geq 1$, and let $\phi \in \mathcal{S}_{s}^{\sigma}\left(\mathbf{R}^{d}\right)$ be fixed. Then the short-time Fourier transform $V_{\phi} f$ of $f \in\left(\mathcal{S}_{s}^{\sigma}\right)^{\prime}\left(\mathbf{R}^{d}\right)$ with respect to the window function $\phi$ is defined by

$$
V_{\phi} f(x, \xi) \equiv \mathscr{F}(f \cdot \overline{\phi(\cdot-x)})(\xi), \quad x, \xi \in \mathbf{R}^{d} .
$$

This definition makes sense as a Gelfand-Shilov distribution [1, Remark $1.5]$.

If $f, \phi \in \mathcal{S}_{s}^{\sigma}\left(\mathbf{R}^{d}\right)$, then

$$
V_{\phi} f(x, \xi)=(2 \pi)^{-\frac{d}{2}} \int f(y) \overline{\phi(y-x)} e^{-i\langle y, \xi\rangle} d y .
$$

Let $s, \sigma>0$, such that $s+\sigma \geq 1$. Let there be given $\phi \in \mathcal{S}_{s}^{\sigma}\left(\mathbf{R}^{d}\right) \backslash 0$, $p, q \in[1, \infty]$ and $\omega \in \mathscr{P}_{E}\left(\mathbf{R}^{2 d}\right)$. Then the modulation space $M_{(\omega)}^{p, q}\left(\mathbf{R}^{d}\right)$ consists of all Gelfand-Shilov distributions $f$ on $\mathbf{R}^{d}$ such that

$$
\|f\|_{M_{(\omega)}^{p, q}} \equiv\left(\int\left(\int\left|V_{\phi} f(x, \xi) \omega(x, \xi)\right|^{p} d x\right)^{q / p} d \xi\right)^{1 / q}<\infty
$$

(with the obvious changes if $p=\infty$ and/or $q=\infty$ ). If $p=q$ we simply write $M_{(\omega)}^{p}$ instead of $M_{(\omega)}^{p, p}$, and if $\omega=1$, then we set $M^{p, q}=M_{(\omega)}^{p, q}$ and $M^{p}=M_{(\omega)}^{p}$.

The spaces $M_{(\omega)}^{p, q}$ are Banach spaces and every $\phi \in M_{(v)}^{r} \backslash 0$ yields an equivalent norm in (1.11) and so $M_{(\omega)}^{p, q}$ is independent on the choice of $\phi \in M_{(v)}^{r}$ [49, Proposition 1.1].

Gelfand-Shilov spaces and their dual spaces can be described as projective or inductive limits of modulation spaces [47, Theorem 3.9]. In particular, we have the following characterization of Gelfand-Shilov spaces by the means of the short-time Fourier transform. We refer to [26] for the proof, see also [36, 40, 47]. 
Proposition 1.8. Let $k \in \mathbf{N}, \sigma=\left(\sigma_{0}, \ldots, \sigma_{k}\right)>0, \mathbf{s}=\left(s_{0}, \ldots, s_{k}\right)>$ 0 , and $\mathbf{d}=d_{1}+\cdots+d_{k}$. Also let $\phi \in \mathcal{S}_{\mathbf{s}}^{\sigma}\left(\mathbf{R}^{\mathbf{d}}\right) \backslash 0$. Then the following is true:

(1) $F \in \mathcal{S}_{\mathbf{s}}^{\sigma}\left(\mathbf{R}^{\mathbf{d}}\right)$ if and only if

$$
\left|V_{\phi} F\left(x_{0}, \ldots, x_{k}, \xi_{0}, \ldots, \xi_{k}\right)\right| \lesssim e^{-r\left(\left|x_{0}\right|^{\frac{1}{s_{0}}}+\cdots+\left|x_{k}\right|^{\frac{1}{s_{k}}}+\left|\xi_{0}\right|^{\frac{1}{\sigma_{0}}}+\cdots+\left|\xi_{k}\right|^{\frac{1}{\sigma_{k}}}\right)}
$$

holds for some $r>0$;

(2) if, in addition, $\phi \in \Sigma_{\mathbf{s}}^{\sigma}\left(\mathbf{R}^{\mathbf{d}}\right) \backslash 0$, then $F \in \Sigma_{\mathbf{s}}^{\sigma}\left(\mathbf{R}^{\mathbf{d}}\right)$ if and only if (1.12) holds for every $r>0$.

1.4. Symbol classes and Pseudo-differential operators. First we introduce function spaces related to symbol classes of the multilinear pseudo-differential operators. We consider $a \in C^{\infty}\left(\mathbf{R}^{d_{0}+\cdots+d_{k}}\right)$ which obey various conditions of the form

$$
\begin{aligned}
\left|\partial_{x}^{\alpha} \partial_{\xi_{1}}^{\beta_{1}} \ldots, \partial_{\xi_{k}}^{\beta_{k}} a\left(x, \xi_{1}, \ldots, \xi_{k}\right)\right| & \\
& \lesssim h^{\left|\alpha+\beta_{1}+\cdots+\beta_{k}\right|} \alpha !^{\sigma} \prod_{j=1}^{k} \beta_{j} !^{!_{j}} \cdot \omega\left(x, \xi_{1}, \ldots, \xi_{k}\right),
\end{aligned}
$$

$w \in \mathscr{P}_{E}\left(\mathbf{R}^{d_{0}+d_{1}+\cdots+d_{k}}\right), \alpha \in \mathbf{N}^{d_{0}}, \beta_{j} \in \mathbf{R}^{d_{j}}, s_{j}, \sigma, h>0, j=1, \ldots, k$.

When $k=1$ we recover the condition (1.14) from [3]. Similarly to [3], for a given $w \in \mathscr{P}_{E}\left(\mathbf{R}^{d_{0}+d_{1}+\cdots+d_{k}}\right)$ and $s_{j}, \sigma, h>0, j=1, \ldots, k$, we consider norms of the form

$$
\begin{aligned}
& \|a\|_{\Gamma_{(\omega)}^{\sigma, \mathbf{s} h}} \equiv \\
& \sup _{\substack{\alpha \in \mathbf{N}^{d_{0}} \\
\beta_{j} \in \mathbf{N}^{d_{j}}}}\left(\sup _{\substack{x \in \mathbf{R}^{d_{0}} \\
\xi_{j} \in \mathbf{R}^{d_{j}}}}\left(\frac{\left|\partial_{x}^{\alpha} \partial_{\xi_{1}}^{\beta_{1}} \ldots, \partial_{\xi_{k}}^{\beta_{k}} a\left(x, \xi_{1}, \ldots, \xi_{k}\right)\right|}{h^{\left|\alpha+\beta_{1}+\cdots+\beta_{k}\right|} \alpha !^{\sigma} \prod_{j=1}^{k} \beta_{j} !^{s_{j}} \cdot \omega\left(x, \xi_{1}, \ldots, \xi_{k}\right)}\right)\right) .
\end{aligned}
$$

More precisely, we are interested in invariance and continuity for bilinear pseudo-differential operators when symbols belong to the following symbol classes.

Definition 1.9. Let there be given $\sigma, s_{j}, h>0, j=1, \ldots, k$ and $\omega \in \mathscr{P}_{E}\left(\mathbf{R}^{d_{0}+d_{1}+\cdots+d_{k}}\right)$, and set $\mathbf{s}=\left(s_{1}, \ldots, s_{k}\right)$.

(1) The set $\Gamma_{(\omega)}^{\sigma, s ; h}\left(\mathbf{R}^{d_{0}+\cdots+d_{k}}\right)$ consists of all $a \in C^{\infty}\left(\mathbf{R}^{d_{0}+\cdots+d_{k}}\right)$ such that

$$
\|a\|_{\Gamma_{(\omega)}^{\sigma, \mathbf{s} h}}<\infty
$$

where the norm $\|\cdot\|_{\Gamma_{(\omega)}^{\sigma, \mathbf{s} ; h}}$ is given by (1.13). 
(2) The sets $\Gamma_{(\omega)}^{\sigma, \mathbf{s}}\left(\mathbf{R}^{d_{0}+\cdots+d_{k}}\right)$ and $\Gamma_{(\omega)}^{\sigma, \mathbf{s} ; 0}\left(\mathbf{R}^{d_{0}+\cdots+d_{k}}\right)$ are given by

$$
\Gamma_{(\omega)}^{\sigma, \mathbf{s}}\left(\mathbf{R}^{d_{0}+\cdots+d_{k}}\right) \equiv \bigcup_{h>0} \Gamma_{(\omega)}^{\sigma, \mathbf{s} ; h}\left(\mathbf{R}^{d_{0}+\cdots+d_{k}}\right)
$$

and

$$
\Gamma_{(\omega)}^{\sigma, \mathbf{s} ; 0}\left(\mathbf{R}^{d_{0}+\cdots+d_{k}}\right) \equiv \bigcap_{h>0} \Gamma_{(\omega)}^{\sigma, \mathbf{s} ; h}\left(\mathbf{R}^{d_{0}+\cdots+d_{k}}\right),
$$

and their topologies are, respectively, the inductive and the projective limit topologies of $\Gamma_{(\omega)}^{\sigma, \mathbf{s} ; h}\left(\mathbf{R}^{d_{0}+\cdots+d_{k}}\right)$ with respect to $h>0$.

As it is common in the theory of ultradifferentiable functions, we say that (the inductive limit) $\Gamma_{(\omega)}^{\sigma, \mathbf{s}}\left(\mathbf{R}^{d_{0}+\cdots+d_{k}}\right)$ is a Roumieu class, and (the projective limit) $\Gamma_{(\omega)}^{\sigma, \mathbf{s} ; 0}\left(\mathbf{R}^{d_{0}+\cdots+d_{k}}\right)$ is a Beurling class of test functions.

Notice that $\Gamma_{(\omega)}^{\sigma, \mathbf{s}}\left(\mathbf{R}^{d_{0}+\cdots+d_{k}}\right)$ and $\Gamma_{(\omega)}^{\sigma, \mathbf{s} ; 0}\left(\mathbf{R}^{d_{0}+\cdots+d_{k}}\right)$ are nontrivial for any $\sigma, s_{j}, h>0, j=1, \ldots, k$. For instance by Proposition 1.7 for any $\omega \in \mathscr{P}_{E}\left(\mathbf{R}^{d_{0}+d_{1}+\cdots+d_{k}}\right)$ there exist a smooth function $\omega_{0} \in \mathscr{P}_{E}\left(\mathbf{R}^{d_{0}+d_{1}+\cdots+d_{k}}\right)$ such that $\omega_{0} \in \Gamma_{(\omega)}^{\sigma, \mathbf{s} ; 0}\left(\mathbf{R}^{d_{0}+\cdots+d_{k}}\right)$.

When $k=1$ we put $\sigma_{1}=\sigma$ and recover the symbol classes $\Gamma_{(\omega)}^{\sigma, s}\left(\mathbf{R}^{d_{0}+d_{1}}\right)$ and $\Gamma_{(\omega)}^{\sigma, s ; 0}\left(\mathbf{R}^{d_{0}+d_{1}}\right)$ considered in $[3]$.

Next we recall some facts on pseudo-differential operators. The pseudodifferential operator $\operatorname{Op}_{t}(a)$ is the linear and continuous operator on $\mathcal{S}\left(\mathbf{R}^{d}\right)$, defined by the formula

$$
\mathrm{Op}_{t}(a) f(x)=\frac{1}{(2 \pi)^{d}} \iint a(x-t(x-y), \xi) f(y) e^{i\langle x-y, \xi\rangle} d y d \xi, \quad x \in \mathbf{R}^{d} .
$$

More generally, the definition of $\mathrm{Op}_{t}(a)$ extends uniquely to $a \in \mathcal{S}^{\prime}\left(\mathbf{R}^{2 d}\right)$, and then $\mathrm{Op}_{t}(a)$ is continuous from $\mathcal{S}\left(\mathbf{R}^{d}\right)$ to $\mathcal{S}^{\prime}\left(\mathbf{R}^{d}\right)$.

Let $\mathbf{t}=\left(t_{1}, t_{2}, \ldots, t_{m}\right) \in[0,1]^{m}$, be such that $\sum_{j=1}^{m} t_{j} \leq 1$, and put $\vec{f}=\left(f_{1}, f_{2}, \ldots, f_{m}\right) \in \mathscr{S}\left(\mathbf{R}^{m d}\right)$. The multilinear pseudo-differential operator $\mathrm{Op}_{\mathbf{t}}(a)$ from $\mathscr{S}\left(\mathbf{R}^{m d}\right)$ to $\mathscr{S}^{\prime}\left(\mathbf{R}^{d}\right)$ is defined by the formula

$$
\mathrm{Op}_{\mathbf{t}}(a) \vec{f}(x)=\frac{1}{(2 \pi)^{2 m d}} \iint e^{-i \psi(x, \mathbf{y}, \xi)} a_{\mathbf{t}}(x, \mathbf{y}, \xi) \prod_{j=1}^{m} f_{j}\left(y_{j}\right) d \mathbf{y} d \xi
$$

where

$$
a_{\mathbf{t}}(x, \mathbf{y}, \xi)=a\left(x+\sum_{j=1}^{m}\left(t_{j} y_{j}-x\right), \xi, \eta\right), \quad x, y_{j}, \xi_{j} \in \mathbf{R}^{d},
$$

and the phase function $\psi$ is defined by

$$
\psi(x, \mathbf{y}, \xi)=\sum_{j=1}^{m}\left\langle y_{j}-x, \xi_{j}\right\rangle, \quad x, y_{j}, \xi_{j} \in \mathbf{R}^{d} .
$$


When $m=2$ we obtain bilinear pseudo-differential operators $\operatorname{Op}_{r, t}(a)$. That is, $\mathrm{Op}_{r, t}(a)$ is the bilinear and continuous operator from $\mathscr{S}\left(\mathbf{R}^{d}\right) \otimes$ $\mathscr{S}\left(\mathbf{R}^{d}\right)$ to $\mathscr{S}^{\prime}\left(\mathbf{R}^{d}\right)$, defined by the formula

$$
\begin{gathered}
\left(\mathrm{Op}_{r, t}(a)(f, g)\right)(x)= \\
(2 \pi)^{-2 d} \iiint \int e^{-i \psi(x, y, z, \xi, \eta)} a_{r, t}(x, y, z, \xi, \eta) f(y) g(z) d y d z d \xi d \eta, x \in \mathbf{R}^{d}
\end{gathered}
$$

where $(r, t) \in[0,1] \times[0,1], r+t \leq 1$,

$$
a_{r, t}(x, y, z, \xi, \eta)=a(x+r(y-x)+t(z-x), \xi, \eta), \quad x, y, z, \xi, \eta \in \mathbf{R}^{d},
$$

and the phase function $\psi$ is defined by

$$
\psi(x, y, z, \xi, \eta)=\langle y-x, \xi\rangle+\langle z-x, \eta\rangle, \quad x, y, z, \xi, \eta \in \mathbf{R}^{d} .
$$

If $r=t=0$, then the definition of $\operatorname{Op}_{0}(a)$ coincides with the definition of bilinear pseudo-differential operators

$$
T_{a}(f, g)(x)=(2 \pi)^{-d} \iint e^{i\langle x, \xi+\eta\rangle} a(x, \xi, \eta) \widehat{f}(\xi) \widehat{g}(\eta) d \xi d \eta, \quad x \in \mathbf{R}^{d},
$$

considered in e.g [7], and the corresponding multilinear extension is studied in [31].

In fact, in Sections 2 and 3 we will consider the action of $\mathrm{Op}_{r, t}(a)$ when restricted to different Gelfand-Shilov spaces, and related unique extension of such operators to Gelfand-Shilov distributions.

We will use the following results about the continuity of linear pseudodifferential operators with symbols in Gevrey-Hörmander classes, and we refer to [3, Theorem 2.1] and [1, Theorem 3.7] respectively, for the proofs.

Proposition 1.10. Let $s, \sigma \geq 1, p, q \in[1, \infty], \omega, \omega_{0} \in \mathscr{P}_{s, \sigma}^{0}\left(\mathbf{R}^{2 d}\right)$, and $a \in \Gamma_{\left(\omega_{0}\right)}^{\sigma, s}\left(\mathbf{R}^{2 d}\right)$. Then $\mathrm{Op}_{t}(a)$ is a continuous operators from $M_{\left(\omega_{0} \omega\right)}^{p, q}\left(\mathbf{R}^{d}\right)$ to $M_{(\omega)}^{p, q}\left(\mathbf{R}^{d}\right)$ for any $t \in[0,1]$.

Note that, in the notation of Definition [1.6 we have $\mathscr{P}_{s, \sigma}^{0}\left(\mathbf{R}^{2 d}\right)=$ $\mathscr{P}_{s_{0}, s_{1}}^{0}\left(\mathbf{R}^{d_{0}+d_{1}}\right)$ when $s_{0}=s, s_{1}=\sigma$ and $d_{1}=d_{2}=d$.

Proposition 1.11. Let $A \in \mathbf{M}(d, R), s, \sigma>0$ be such that $s+\sigma \geq 1$, $\omega \in \mathscr{P}_{s, \sigma}^{0}\left(\mathbf{R}^{2 d}\right)$ and let $a \in \Gamma_{0}^{\sigma, s ; h}\left(\mathbf{R}^{2 d}\right)$ for some $h>0$. Then $\mathrm{Op}_{A}(a)$ is continuous on $\mathcal{S}_{s}^{\sigma}\left(\mathbf{R}^{d}\right)$ and on $\left(\mathcal{S}_{s}^{\sigma}\right)^{\prime}\left(\mathbf{R}^{d}\right)$.

\section{Characterization AND INVARIANCE PROPERTY FOR BILINEAR PSEUDO-DIFFERENTIAL OPERATORS}

Our aim in this section is to show that $\Gamma_{(\omega)}^{\sigma, s_{1}, s_{2}}\left(\mathbf{R}^{3 d}\right)$ and $\Gamma_{(\omega)}^{\sigma, s_{1}, s_{2} ; 0}\left(\mathbf{R}^{3 d}\right)$ can be characterized in terms of estimates of short-time Fourier transforms and modulation spaces. This is done in subsection 2.2. We refer 
to [1,48 for similar results related to "standard" pseudo-differential operators. As a preparation, we show that $\mathrm{Op}_{r, t}(a)$ is independent of the choice of $r$ and $t$, which gives the invariance property for bilinear operators, Theorem 2.2. The counterpart of Theorem 2.2 for "standard" pseudo-differential is proved in e.g. [1, 3, 48]. The key tools we employ to achieve the desired characterizations and invariance properties in this section are mapping results for exponentials of certain linear operators, similar to the typical ones often appearing in the "usual" Weyl-Hörmander calculus, whose description is given here below.

\subsection{Mapping properties of exponential-type operators on Gel-} fand-Shilov spaces. For the study of mapping properties of the operator $e^{-i\left\langle r D_{\xi}+t D_{\eta}, D_{x}\right\rangle}$ we need the following auxiliary result. $\operatorname{By} \mathbf{M}(d, \mathbf{R})$ we denote the set of all $d \times d$-matrices with entries in $\mathbf{R}$.

Lemma 2.1. Let $A, B \in \mathbf{M}(d, \mathbf{R})$ and $a \in \mathscr{S}\left(\mathbf{R}^{3 d}\right)$. Then

$$
\left(\mathscr{F}_{2,3}^{-1}\left(e^{i\left\langle A D_{\xi}+B D_{\eta}, D_{x}\right\rangle} a\right)\right)(x+A y+B z, y, z)=\left(\mathscr{F}_{2,3}^{-1} a\right)(x, y, z),
$$

$x, y, z \in \mathbf{R}^{d}$.

Proof. Throughout the proof, the integrals are observed as either Fourier transforms or inverse Fourier transforms of appropriate distributions. The left-hand side of (2.1) is given by

$$
\begin{aligned}
& \left(\mathscr{F}_{2,3}^{-1}\left(e^{i\left\langle A D_{\xi}+B D_{\eta}, D_{x}\right\rangle} a\right)\right)(x+A y+B z, y, z) \\
& \left.\quad=\iint e^{i(\langle y, \xi\rangle+\langle z, \eta\rangle)}\left(e^{i\left\langle A D_{\xi}+B D_{\eta}, D_{x}\right\rangle} a\right)\right)(x+A y+B z, \xi, \eta) d \xi d \eta
\end{aligned}
$$

and

$$
\begin{aligned}
& \left.\left(e^{i\left\langle A D_{\xi}+B D_{\eta}, D_{x}\right\rangle} a\right)\right)(x+A y+B z, \xi, \eta) \\
& =\iiint e^{i\left(\langle x+A y+B z, \zeta\rangle+\left\langle y_{1}, \xi\right\rangle+\left\langle z_{1}, \eta\right\rangle\right)} e^{i\left(\left\langle A y_{1}+B z_{1}, \zeta\right\rangle\right)} \widehat{a}\left(\zeta, y_{1}, z_{1}\right) d \zeta d y_{1} d z_{1},
\end{aligned}
$$

where

$$
\widehat{a}\left(\zeta, y_{1}, z_{1}\right)=\iiint e^{-i\left(\left\langle x_{1}, \zeta\right\rangle+\left\langle y_{1}, \xi_{1}\right\rangle+\left\langle z_{1}, \eta_{1}\right\rangle\right)} a\left(x_{1}, \xi_{1}, \eta_{1}\right) d x_{1} d \xi_{1} d \eta_{1},
$$

$x_{1}, \xi_{1}, \eta_{1} \in \mathbf{R}^{d}$. Let $\Psi \equiv \Psi\left(x, x_{1}, y, y_{1}, z, z_{1}, \zeta, \xi, \eta, \xi_{1}, \eta_{1}\right)$ be given by

$$
\begin{aligned}
\Psi=\left\langle y+y_{1}, \xi\right\rangle+ & \left\langle z+z_{1}, \eta\right\rangle+\langle x+A y+B z, \zeta\rangle \\
& +\left(\left\langle A y_{1}+B z_{1}, \zeta\right\rangle\right)-\left(\left\langle x_{1}, \zeta\right\rangle+\left\langle y_{1}, \xi_{1}\right\rangle+\left\langle z_{1}, \eta_{1}\right\rangle\right),
\end{aligned}
$$


$x, x_{1}, y, y_{1}, z, z_{1}, \zeta, \xi, \eta, \xi_{1}, \eta_{1} \in \mathbf{R}^{d}$. It follows that

$$
\begin{aligned}
& \left(\mathscr{F}_{2,3}^{-1}\left(e^{i\left\langle A D_{\xi}+B D_{\eta}, D_{x}\right\rangle} a\right)\right)(x+A y+B z, y, z) \\
& =\iiint \iiint \iint e^{i \Psi\left(x, x_{1}, y, y_{1}, z, z_{1}, \zeta, \xi, \eta, \xi_{1}, \eta_{1}\right)} \\
& \times a\left(x_{1}, \xi_{1}, \eta_{1}\right) d x_{1} d \xi_{1} d \eta_{1} d \zeta d y_{1} d z_{1} d \xi d \eta, \quad x, y, z \in \mathbf{R}^{d} .
\end{aligned}
$$

Since

$$
\int e^{i\left\langle y+y_{1}, \xi\right\rangle} d \xi=\delta\left(y+y_{1}\right), \quad \text { and } \int e^{i\left\langle z+z_{1}, \eta\right\rangle} d \eta=\delta\left(z+z_{1}\right),
$$

where $\delta$ is the Dirac delta distribution, it follows that (2.2) reduces to

$$
\begin{aligned}
\iiint \int e^{i\left(\langle x, \zeta\rangle-\left\langle x_{1}, \zeta\right\rangle+\left\langle y, \xi_{1}\right\rangle+\left\langle z, \eta_{1}\right\rangle\right)} a\left(x_{1}, \xi_{1}, \eta_{1}\right) d \zeta d x_{1} d \xi_{1} d \eta_{1} \\
=\iint e^{i\left(\left\langle y, \xi_{1}\right\rangle+\left\langle z, \eta_{1}\right\rangle\right)} a\left(x, \xi_{1}, \eta_{1}\right) d \xi_{1} d \eta_{1}=\left(\mathscr{F}_{2,3}^{-1} a\right)(x, y, z),
\end{aligned}
$$

and the claim follows.

Next we show some mapping properties of the operator $e^{-i\left\langle r D_{\xi}+t D_{\eta}, D_{x}\right\rangle}$ which are an important ingredient in our analysis.

Theorem 2.2. Let $s_{j}, \sigma_{j}, j=1,2,3$, be such that

$$
s_{j}+\sigma_{j} \geq 1, \quad 0<s_{2}, s_{3} \leq s_{1}, \quad \text { and } \quad 0<\sigma_{1} \leq \sigma_{2}, \sigma_{3}
$$

and let $r, t \in[0,1]$ be such that $r+t \leq 1$. Then the following is true:

(1) $e^{-i\left\langle r D_{\xi}+t D_{\eta}, D_{x}\right\rangle}$ on $\mathscr{S}\left(\mathbf{R}^{3 d}\right)$ restricts to a homeomorphism on $\mathcal{S}_{s_{1}, \sigma_{2}, \sigma_{3}}^{\sigma_{1}, s_{2}, s_{3}}\left(\mathbf{R}^{3 d}\right)$, and extends uniquely to a homeomorphism on $\left(\mathcal{S}_{s_{1}, \sigma_{2}, \sigma_{3}}^{\sigma_{1}, s_{2}, s_{3}}\right)^{\prime}\left(\mathbf{R}^{3 d}\right)$;

(2) if in addition $\left(s_{j}, \sigma_{j}\right) \neq\left(\frac{1}{2}, \frac{1}{2}\right), j=1,2,3$, then $e^{-i\left\langle r D_{\xi}+t D_{\eta}, D_{x}\right\rangle}$ on $\mathscr{S}\left(\mathbf{R}^{3 d}\right)$ restricts to a homeomorphism on $\sum_{s_{1}, \sigma_{2}, \sigma_{3}}^{\sigma_{1}, s_{2}, s_{3}}\left(\mathbf{R}^{3 d}\right)$, and extends uniquely to a homeomorphism on $\left(\Sigma_{s_{1}, \sigma_{2}, \sigma_{3}}^{\sigma_{1}, s_{2}, s_{3}}\right)^{\prime}\left(\mathbf{R}^{3 d}\right)$.

Proof. We only prove (1) and leave (2) for the reader.

Let $a \in \mathscr{S}\left(\mathbf{R}^{3 d}\right)$ and let $U_{r, t}$ be the map given by

$$
\left(U_{r, t} F\right)(x, y)=F(x-r y-t z, y, z), \quad x, y \in \mathbf{R}^{d} .
$$

By Lemma 2.1, we have

$$
\left(\mathscr{F}_{2,3}^{-1}\left(e^{i\left\langle r D_{\xi}+t D_{\eta}, D_{x}\right\rangle} a\right)\right)(x+r y+t z, y, z)=\left(\mathscr{F}_{2,3}^{-1} a\right)(x, y, z), \quad x, y, z \in \mathbf{R}^{d},
$$

wherefrom $e^{i\left\langle r D_{\xi}+t D_{\eta}, D_{x}\right\rangle}=\mathscr{F}_{2,3} \circ U_{r, t} \circ \mathscr{F}_{2,3}^{-1}$. Therefore it only remains to show that the mapping $U_{r, t}$ is continuous on $\mathcal{S}_{s_{1}, s_{2}, s_{3}}^{\sigma_{1}, \sigma_{2},}$

Since the Fourier transform with respect to the $2^{\text {nd }}$ and $3^{\text {rd }}$ variables switches between the corresponding decay and regularity properties on Gelfand-Shilov spaces we consider $G=U_{r, t} F$, where $F \in \mathcal{S}_{s_{1}, \sigma_{2}, \sigma_{3}}^{\sigma_{1}, s_{2}, s_{3}}$. Then $G(x, y, z)=F(x-r y-t z, y, z)$ and $\widehat{G}(\zeta, \xi, \eta)=\widehat{F}(\zeta, \zeta+r \xi, \zeta+t \eta)$, 
$x, y, z, \zeta, \xi, \eta \in \mathbf{R}^{d}$. In view of Proposition 1.4 and from the assumptions on $s_{j}$ and $\sigma_{j}$, it follows that there exist constants $c, r_{0}>0$, where $c$ depends on $r, t, s_{j}$ and $\sigma_{j}$ only, such that

$$
\begin{aligned}
|G(x, y, z)|=\mid & F(x-r y-t z, y, z) \mid \\
& \lesssim e^{-r_{0}\left(|x-r y-t z|^{\frac{1}{s_{1}}}+|y|^{\frac{1}{s_{2}}}+|z|^{\frac{1}{s_{3}}}\right)} \lesssim e^{-c r_{0}\left(|x|^{\frac{1}{s_{1}}}+|y|^{\frac{1}{s_{2}}}+|z|^{\frac{1}{s_{3}}}\right)},
\end{aligned}
$$

$x, y, z \in \mathbf{R}^{d}$, and

$$
\begin{aligned}
& |\widehat{G}(\zeta, \xi, \eta)|=|\widehat{F}(\zeta, \zeta+r \xi, \zeta+t \eta)| \\
& \quad \lesssim e^{-r\left(|\zeta|^{\frac{1}{\sigma_{1}}}+|\zeta+r \xi|^{\frac{1}{\sigma_{2}}}|\zeta+t \eta|^{\frac{1}{\sigma_{3}}}\right)} \lesssim e^{-c r\left(|\zeta|^{\frac{1}{\sigma_{1}}}+|\xi|^{\frac{1}{\sigma_{2}}}+|\eta|^{\frac{1}{\sigma_{3}}}\right)}
\end{aligned}
$$

$\zeta, \xi, \eta \in \mathbf{R}^{d}$. The result follows since by Proposition 1.5 the topology in $\mathcal{S}_{s_{1}, s_{2}, s_{3}}^{\sigma_{1}, \sigma_{2}, \sigma_{3}}\left(\mathbf{R}^{3 d}\right)$ can be defined by the above estimates.

Corollary 2.3. Let $s, \sigma>0$ be such that $s+\sigma \geq 1$ and $\sigma \leq s$. Then $e^{-i\left\langle r D_{\xi}+t D_{\eta}, D_{x}\right\rangle}$ is a homeomorphism on $\mathcal{S}_{s}^{\sigma}\left(\mathbf{R}^{3 d}\right), \Sigma_{s}^{\sigma}\left(\mathbf{R}^{3 d}\right),\left(\mathcal{S}_{s}^{\sigma}\right)^{\prime}\left(\mathbf{R}^{3 d}\right)$ and on $\left(\Sigma_{s}^{\sigma}\right)^{\prime}\left(\mathbf{R}^{3 d}\right)$.

Next we study an invariance property of bilinear pseudo-differential operators $\mathrm{Op}_{r, t}(a)$ given by (1.14). More precisely, it can be shown that for every Gelfand-Shilov distribution $a$ there is a unique distribution $b$ in the same Gelfand-Shilov class such that $\mathrm{Op}_{r_{1}, t_{1}}(a)=\mathrm{Op}_{r_{2}, t_{2}}(b)$, when $r_{j}, t_{j} \in[0,1]$ and $r_{j}+t_{j} \leq 1$. The following result, which explains the relation between such $a$ and $b$, follows from Theorem 2.2 when the conditions in (2.3) are fulfilled. We give an independent proof in Appendix A

Proposition 2.4. Let $r_{j}, t_{j} \in[0,1]$ be such that $r_{j}+t_{j} \leq 1$, and let $a, b \in\left(\mathcal{S}_{s_{1}, \sigma_{2}, \sigma_{3}}^{\sigma_{1}, s_{2}, s_{3}}\right)^{\prime}\left(\mathbf{R}^{3 d}\right)$, where $s_{j}, \sigma_{j}>0$, and $s_{j}+\sigma_{j} \geq 1, j=1,2,3$. Then

$$
\begin{aligned}
\mathrm{Op}_{r_{1}, t_{1}}(a) & =\mathrm{Op}_{r_{2}, t_{2}}(b) \\
& \Leftrightarrow \\
e^{-i\left\langle r_{1} D_{\xi}+t_{1} D_{\eta}, D_{x}\right\rangle} a(x, \xi, \eta) & =e^{-i\left\langle r_{2} D_{\xi}+t_{2} D_{\eta}, D_{x}\right\rangle} b(x, \xi, \eta), \quad x, \xi, \eta \in \mathbf{R}^{d} .
\end{aligned}
$$

Note that the latter equality in (2.4) makes sense since it is equivalent to

$$
e^{-i\left\langle r_{1} y+t_{1} z, \zeta\right\rangle} \widehat{a}(\zeta, y, z)=e^{-i\left\langle r_{2} y+t_{2} z, \zeta\right\rangle} \widehat{b}(\zeta, y, z), \quad \zeta, y, z \in \mathbf{R}^{d} .
$$

Moreover, by using the similar arguments as in e. g. [1, 9, 50], it can be shown that the map $a \mapsto e^{-i\langle r y+t z, \zeta\rangle} a$ is continuous on $\left(\mathcal{S}_{s_{1}, \sigma_{2}, \sigma_{3}}^{\sigma_{1}, s_{2}, s_{3}}\right)^{\prime}\left(\mathbf{R}^{3 d}\right)$.

The following corollary is a consequence of [9, Theorem 4.6] and Proposition 2.4. 
Corollary 2.5. Let $s_{j}, \sigma_{j}>0$ be such that $s_{j}+\sigma_{j} \geq 1,\left(s_{j}, \sigma_{j}\right) \neq\left(\frac{1}{2}, \frac{1}{2}\right)$, $j=1,2,3$ and $r_{j}, t_{j} \in[0,1]$ be such that $r_{j}+t_{j} \leq 1, j=1,2$. If $a, b \in\left(\Sigma_{s_{1}, \sigma_{2}, \sigma_{3}}^{\sigma_{1}, s_{2}, s_{3}}\right)^{\prime}\left(\mathbf{R}^{3 d}\right)$, are such that $\mathrm{Op}_{r_{1}, t_{1}}(a)=\mathrm{Op}_{r_{2}, t_{2}}(b)$ Then

$$
a \in \Gamma_{(\omega)}^{\sigma_{1}, s_{2}, s_{3} ; 0}\left(\mathbf{R}^{3 d}\right) \quad \Leftrightarrow \quad b \in \Gamma_{(\omega)}^{\sigma_{1}, s_{2}, s_{3} ; 0}\left(\mathbf{R}^{3 d}\right)
$$

and

$$
a \in \Gamma_{(\omega)}^{\sigma_{1}, s_{2}, s_{3}}\left(\mathbf{R}^{3 d}\right) \quad \Leftrightarrow \quad b \in \Gamma_{(\omega)}^{\sigma_{1}, s_{2}, s_{3}}\left(\mathbf{R}^{3 d}\right),
$$

for any given $\omega \in \mathscr{P}_{E}\left(\mathbf{R}^{3 d}\right)$.

Passages between different kinds of pseudo-differential calculi have been considered before [27, 50]. On the other hand, for the bilinear pseudo-differential calculi, it seems that the representation $a \mapsto \mathrm{Op}_{r, t}(a)$ for $(r, t) \in[0,1] \times[0,1]$ such that $r+t \leq 1$, has not been considered so far.

2.2. Gevrey-type symbol classes characterizations. . Our first result concerns the Roumieu case of symbols in $\Gamma_{(\omega)}^{\sigma, s, s}\left(\mathbf{R}^{3 d}\right)$. It can be deduced from [9, Proposition 4.3], see also [1, Proposition 2.4]. For the sake of completeness, we give the proof.

Proposition 2.6. Let $s_{j}, \sigma_{j}>0, j=1,2,3$, be such that the conditions in (2.3) hold, let $\omega \in \mathscr{P}_{s_{1}, \sigma_{2}, \sigma_{3}}^{0}\left(\mathbf{R}^{3 d}\right)$ and let a be a Gelfand-Shilov distribution on $\mathbf{R}^{3 d}$.

Then the following conditions are equivalent:

(1) $a \in \Gamma_{(\omega)}^{\sigma_{1}, s_{2}, s_{3}}\left(\mathbf{R}^{3 d}\right)$, that is, $a \in C^{\infty}\left(\mathbf{R}^{3 d}\right)$ and $\left|\partial_{x}^{\alpha} \partial_{\xi}^{\beta} \partial_{\eta}^{\gamma} a(x, \xi, \eta)\right| \lesssim h^{|\alpha+\beta+\gamma|} \alpha !^{\sigma_{1}} \beta !^{s_{2}} \gamma !^{s_{3}} \omega(x, \xi, \eta), \quad x, \xi, \eta \in \mathbf{R}^{d}$, for every $\alpha, \beta, \gamma \in \mathbf{N}^{d}$ and some $h>0$;

(2) For every $\phi \in \mathcal{S}_{s_{1}, \sigma_{2}, \sigma_{3}}^{\sigma_{1}, s_{2}, s_{3}}\left(\mathbf{R}^{3 d}\right) \backslash 0$, there exist constants $h, R>0$ such that for every $\alpha, \beta, \gamma \in \mathbf{N}^{d}$,

$$
\begin{aligned}
& \left|\partial_{x}^{\alpha} \partial_{\xi}^{\beta} \partial_{\eta}^{\gamma}\left(e^{i(\langle x, \zeta\rangle+\langle y, \xi\rangle+\langle z, \eta\rangle)} V_{\phi} a(x, \xi, \eta, \zeta, y, z)\right)\right| \\
& \quad \lesssim h^{|\alpha+\beta+\gamma|} \alpha !^{\sigma_{1}} \beta !^{s_{2}} \gamma !^{s_{3}} \omega(x, \xi, \eta) e^{-R\left(|\zeta|^{\frac{1}{\sigma_{1}}}+|y|^{\frac{1}{s_{2}}}+|z|^{\frac{1}{s_{3}}}\right)}, \\
& x, \xi, \eta, \zeta, y, z \in \mathbf{R}^{d} .
\end{aligned}
$$

(3) For every $\phi \in \mathcal{S}_{s_{1}, \sigma_{2}, \sigma_{3}}^{\sigma_{1}, s_{2}, s_{3}}\left(\mathbf{R}^{3 d}\right) \backslash 0$, there exist a constant $R>0$ such that

$\left|V_{\phi} a(x, \xi, \eta, \zeta, y, z)\right| \lesssim \omega(x, \xi, \eta) e^{-R\left(|\zeta|^{\frac{1}{\sigma_{1}}}+|y|^{\frac{1}{s_{2}}}+|z|^{\frac{1}{s_{3}}}\right)}, \quad x, \xi, \eta, \zeta, y, z \in \mathbf{R}^{d}$.

Proof. That (2) implies (3) is immediate, since (2.5) is equal to (2.6) when $\alpha=\beta=\gamma=0$. 
Let $X=(x, \xi, \eta), Y=\left(x_{1}, \xi_{1}, \eta_{1}\right), Z=(\zeta, y, z) \in \mathbf{R}^{3 d}$, and set

$$
F_{a}(X, Y)=a(X+Y) \phi(Y)=a\left(x+x_{1}, \xi+\xi_{1}, \eta+\eta_{1}\right) \phi\left(x_{1}, \xi_{1}, \eta_{1}\right) .
$$

Assume that (1) holds true. By the Leibniz rule, (1.10) and Proposition 1.5 we obtain

$$
\begin{aligned}
\left|\partial_{x}^{\alpha} \partial_{\xi}^{\beta} \partial_{\eta}^{\gamma} F_{a}\left(x, \xi, \eta, x_{1}, \xi_{1}, \eta_{1}\right)\right| & \\
& \lesssim h^{|\alpha+\beta+\gamma|} \alpha !^{\sigma_{1}} \beta !^{s_{2}} \gamma !^{s_{3}} \omega(x, \xi, \eta) e^{-R\left(\left|x_{1}\right|^{\frac{1}{s_{1}}}+\left|\xi_{1}\right|^{\frac{1}{\sigma_{2}}}+\left|\eta_{1}\right|^{\frac{1}{\sigma_{3}}}\right)},
\end{aligned}
$$

$x, \xi, \eta, x_{1}, \xi_{1}, \eta_{1} \in \mathbf{R}^{d}$, for some constants $h, R>0$.

It follows that the set

$$
\left\{G_{a, h, X}(Y) \mid G_{a, h, x, \xi, \eta}\left(x_{1}, \xi_{1}, \eta_{1}\right)=\frac{\partial_{x}^{\alpha} \partial_{\xi}^{\beta} \partial_{\eta}^{\gamma} F_{a}\left(x, \xi, \eta, x_{1}, \xi_{1}, \eta_{1}\right)}{h^{|\alpha+\beta+\gamma|} \alpha !^{\sigma_{1}} \beta !^{s_{2}} \gamma !^{s_{3}} \omega(x, \xi, \eta)}\right\}
$$

is bounded in $\mathcal{S}_{s_{1}, \sigma_{2}, \sigma_{3}}^{\sigma_{1}, s_{2}, s_{3}}\left(\mathbf{R}^{3 d}\right)$. If $\mathscr{F}_{2} F_{a}$ denotes the partial Fourier transform of $F_{a}(X, Y)$ with respect to the $Y$-variable, then we get

$$
\begin{aligned}
\mid \partial_{x}^{\alpha} \partial_{\xi}^{\beta} \partial_{\eta}^{\gamma}\left(\mathscr{F}_{2} F_{a}\right) & (x, \xi, \eta, \zeta, y, z) \mid \\
& \lesssim h^{|\alpha+\beta+\gamma|} \alpha !^{\mid \sigma_{1}} \beta !^{s_{2}} \gamma !^{s_{3}} \omega(x, \xi, \eta) e^{-R\left(|y|^{\frac{1}{\sigma_{1}}}+|z|^{\frac{1}{s_{2}}}+|\zeta|^{\frac{1}{s_{3}}}\right)},
\end{aligned}
$$

$x, \xi, \eta, x_{1}, \xi_{1}, \eta_{1} \in \mathbf{R}^{d}$, for some constants $h, R>0$. This, together with the Leibnitz rule applied to $\partial_{x}^{\alpha} \partial_{\xi}^{\beta} \partial_{\eta}^{\gamma}\left(e^{i(\langle x, \zeta\rangle+\langle y, \xi\rangle+\langle z, \eta\rangle)} V_{\phi} a(x, \xi, \eta, \zeta, y, z)\right)$ gives (2).

Assume now that (3) holds. By the inversion formula we get

$$
a(X)=\frac{(2 \pi)^{-\frac{3 d}{2}}}{\|\phi\|_{L^{2}}^{2}} \iint V_{\phi} a(Y, Z) \phi(X-Y) e^{i\langle X, Z\rangle} d Y d Z, \quad X \in \mathbf{R}^{3 d}
$$

in the weak sense. Since $\phi \in \mathcal{S}_{s_{1}, \sigma_{2}, \sigma_{3}}^{\sigma_{1}, s_{2}, s_{3}}\left(\mathbf{R}^{3 d}\right)$ we notice that

$$
(X, Y, Z) \mapsto V_{\phi} a(Y, Z) \phi(X-Y) e^{i\langle X, Z\rangle}
$$

is a smooth map, and

$$
(Y, Z) \mapsto Z^{\boldsymbol{\alpha}} V_{\phi} a(Y, Z) \partial^{\boldsymbol{\beta}} \phi(X-Y) e^{i\langle X, Z\rangle}
$$

is an integrable function for every $X \in \mathbf{R}^{3 d}, \boldsymbol{\alpha}=\left(\alpha_{1}, \alpha_{2}, \alpha_{3}\right) \in \mathbf{N}^{3 d}$, and $\boldsymbol{\beta}=\left(\beta_{1}, \beta_{2}, \beta_{3}\right) \in \mathbf{N}^{3 d}$ in view of (3). Hence the derivatives of $a$ in 
(2.7) satisfy the following estimates:

$$
\begin{gathered}
\left|\partial^{\boldsymbol{\alpha}} a(X)\right| \leq \sum_{\boldsymbol{\beta} \leq \boldsymbol{\alpha}}\left(\begin{array}{c}
\boldsymbol{\alpha} \\
\boldsymbol{\beta}
\end{array}\right) \iint\left|Z^{\boldsymbol{\beta}} V_{\phi} a(Y, Z)\left(\partial^{\boldsymbol{\alpha}-\boldsymbol{\beta}} \phi\right)(X-Y)\right| d Y d Z \\
\lesssim \sum_{\boldsymbol{\beta} \leq \boldsymbol{\alpha}}\left(\begin{array}{c}
\boldsymbol{\alpha} \\
\boldsymbol{\beta}
\end{array}\right) \iint\left|Z^{\boldsymbol{\beta}} \omega(Y) e^{-R\left(|\zeta|^{\frac{1}{\sigma_{1}}}+|y|^{\frac{1}{s_{2}}}+|z|^{\frac{1}{s_{3}}}\right)}\left(\partial^{\boldsymbol{\alpha}-\boldsymbol{\beta}} \phi\right)(X-Y)\right| d Y d Z \\
\lesssim \sum_{\boldsymbol{\beta} \leq \boldsymbol{\alpha}}\left(\begin{array}{c}
\boldsymbol{\alpha} \\
\boldsymbol{\beta}
\end{array}\right) h_{2}^{|\boldsymbol{\alpha}-\boldsymbol{\beta}|}\left(\alpha_{1}-\beta_{1}\right) !^{\sigma_{1}}\left(\alpha_{2}-\beta_{2}\right) !^{s_{2}}\left(\alpha_{3}-\beta_{3}\right) !^{s_{3}} \\
\times \iint\left|Z^{\boldsymbol{\beta}}\right| e^{-R\left(|\zeta|^{\frac{1}{\sigma_{1}}}+|y|^{\frac{1}{s_{2}}}+|z|^{\frac{1}{s_{3}}}\right)} \omega(Y) e^{-h_{1}\left(\left|x-x_{1}\right|^{\frac{1}{s_{1}}}+\left|\xi-\xi_{1}\right|^{\frac{1}{\sigma_{2}}}+\left|\eta-\eta_{1}\right|^{\frac{1}{\sigma_{3}}}\right)} d Y d Z,
\end{gathered}
$$

$X \in \mathbf{R}^{3 d}$, for some constants $h_{1}, h_{2}>0$, and we used Lemma 1.2. For any $\boldsymbol{\beta} \in \mathbf{N}^{3 d}, \sigma, s_{j}>0$ such that $s_{j}+\sigma \geq 1, j=1,2$, and $h_{2}, R>0$, it holds

$$
\left|\zeta^{\beta_{1}} y^{\beta_{2}} z^{\beta_{3}} e^{-R\left(|\zeta|^{\frac{1}{\sigma}}+|y|^{\frac{1}{s}}+|z|^{\frac{1}{s}}\right)}\right| \lesssim h_{2}^{|\boldsymbol{\beta}|} \beta_{1} !^{\sigma_{1}} \beta_{2} !^{s_{2}} \beta_{3} !^{s_{3}} e^{-\frac{R}{2}\left(|\zeta|^{\frac{1}{\sigma_{1}}}+|y|^{\frac{1}{s_{2}}}+|z|^{\frac{1}{s_{3}}}\right)},
$$

$\zeta, y, z \in \mathbf{R}^{d}$, so that

$$
\begin{aligned}
& \left|\partial^{\boldsymbol{\alpha}} a(X)\right| \\
& \lesssim h_{2}^{|\boldsymbol{\alpha}|} \sum_{\boldsymbol{\beta} \leq \boldsymbol{\alpha}}\left(\begin{array}{c}
\boldsymbol{\alpha} \\
\boldsymbol{\beta}
\end{array}\right)\left(\beta_{1} !\left(\alpha_{1}-\beta_{1}\right) !\right)^{\sigma_{1}}\left(\beta_{2} !\left(\alpha_{2}-\beta_{2}\right) !\right)^{s_{2}}\left(\beta_{3} !\left(\alpha_{3}-\beta_{3}\right) !\right)^{s_{3}} \\
& \times \iint e^{-\frac{R}{2}\left(|\zeta|^{\frac{1}{\sigma_{1}}}+|y|^{\frac{1}{s_{2}}}+|z|^{\frac{1}{s_{3}}}\right)} \omega(Y) e^{-h_{1}\left(\left|x-x_{1}\right|^{\frac{1}{s_{1}}}+\left|\xi-\xi_{1}\right|^{\frac{1}{\sigma_{2}}}+\left|\eta-\eta_{1}\right|^{\frac{1}{\sigma_{3}}}\right)} d Y d Z \\
& \lesssim\left(2 h_{2}\right)^{|\boldsymbol{\alpha}|} \alpha_{1} !^{\sigma_{1}} \alpha_{2} !^{s_{2}} \alpha_{3} !^{l_{3}} \\
& \times \int \omega(X+(Y-X)) e^{-h_{1}\left(\left|x-x_{1}\right|^{\frac{1}{s_{1}}}+\left|\xi-\xi_{1}\right|^{\frac{1}{\sigma_{2}}}+\left|\eta-\eta_{1}\right|^{\frac{1}{\sigma_{3}}}\right)} d Y, \quad X \in \mathbf{R}^{3 d} .
\end{aligned}
$$

Since $\omega \in \mathscr{P}_{s_{1}, \sigma_{2}, \sigma_{3}}^{0}$, that is (1.10) holds for every $r>0$, by choosing $r \in\left(0, h_{1} / 2\right)$, from (2.8) it follows that

$$
\left|\partial^{\boldsymbol{\alpha}} a(X)\right| \lesssim\left(2 h_{2}\right)^{|\boldsymbol{\alpha}|} \alpha_{1} !^{\sigma_{1}} \alpha_{2} !^{s_{2}} \alpha_{3} !^{s_{3}} \omega(X), \quad X \in \mathbf{R}^{3 d},
$$

for some constant $h_{2}>0$ (and we conclude that (2.7) holds also in the pointwise sense). Therefore (3) implies (1) and the result follows.

The Beurling case follows by similar arguments as in the proof of Proposition 2.6.

Proposition 2.7. Let $s_{j}, \sigma_{j}>0,\left(s_{j}, \sigma_{j}\right) \neq\left(\frac{1}{2}, \frac{1}{2}\right), j=1,2,3$, and let the conditions in (2.3) hold. Also, let $\omega \in \mathscr{P}_{s_{1}, \sigma_{2}, \sigma_{3}}\left(\mathbf{R}^{3 d}\right)$ and let a be a Gelfand-Shilov distribution on $\mathbf{R}^{3 d}$. Then the following conditions are equivalent: 
(1) a in $\in \Gamma_{(\omega)}^{\sigma_{1}, s_{2}, s_{3} ; 0}\left(\mathbf{R}^{3 d}\right)$, i.e. $a \in C^{\infty}\left(\mathbf{R}^{3 d}\right)$ and $\left|\partial_{x}^{\alpha} \partial_{\xi}^{\beta} \partial_{\eta}^{\gamma} a(x, \xi, \eta)\right| \lesssim h^{|\alpha+\beta+\delta|} \alpha !^{\sigma_{1}} \beta !^{s_{2}} \gamma !^{s_{3}} \omega(x, \xi, \eta), \quad x, \xi, \eta \in \mathbf{R}^{d}$, for every $\alpha, \beta, \gamma \in \mathbf{N}^{d}$ and for every $h>0$;

(2) For every $\phi \in \Sigma_{s_{1}, \sigma_{2}, \sigma_{3}}^{\sigma_{1}, s_{2}, s_{3}}\left(\mathbf{R}^{3 d}\right) \backslash 0, h, R>0$ and $\alpha, \beta, \gamma \in \mathbf{N}^{d}$, it holds

$$
\begin{aligned}
&\left|\partial_{x}^{\alpha} \partial_{\xi}^{\beta} \partial_{\eta}^{\gamma}\left(e^{i(\langle x, \zeta\rangle+\langle y, \xi\rangle+\langle z, \eta\rangle)} V_{\phi} a(x, \xi, \eta, \zeta, y, z)\right)\right| \\
& \lesssim h^{|\alpha+\beta+\gamma|} \alpha !^{\sigma_{1}} \beta !^{s_{2}} \gamma !^{s_{3}} \omega(x, \xi, \eta) e^{-R\left(|\zeta|^{\frac{1}{\sigma_{1}}}+|y|^{\frac{1}{s_{2}}}+|z|^{\frac{1}{s_{3}}}\right)},
\end{aligned}
$$$$
x, \xi, \eta, \zeta, y, z \in \mathbf{R}^{d} .
$$

(3) For every $\phi \in \Sigma_{s_{1}, \sigma_{2}, \sigma_{3}}^{\sigma_{1}, s_{2}, s_{3}}\left(\mathbf{R}^{3 d}\right) \backslash 0$, and $R>0$ it holds

$\left|V_{\phi} a(x, \xi, \eta, \zeta, y, z)\right| \lesssim \omega(x, \xi, \eta) e^{-R\left(|\zeta|^{\frac{1}{\sigma_{1}}}+|y|^{\frac{1}{s_{2}}}+|z|^{\frac{1}{s_{3}}}\right)}, \quad x, \xi, \eta, \zeta, y, z \in \mathbf{R}^{d}$.

In the next result we consider the Beurling case. It gives a description of the symbol class $\Gamma_{(\omega)}^{\sigma_{1}, s_{2}, s_{3} ; 0}\left(\mathbf{R}^{3 d}\right)$ in terms of modulation spaces $M_{\left(1 / \omega_{R}\right)}^{\infty, q}\left(\mathbf{R}^{3 d}\right)$ for $q \in[1, \infty]$ and $\omega_{R}$ defined in (2.9) below. To prove Proposition 2.8 we follow arguments analogous to those used in the proofs of [1, Proposition 3.5] and [9, Proposition 4.4].

Proposition 2.8. Let $R>0, q \in[1, \infty], s_{j}, \sigma_{j}>0,\left(s_{j}, \sigma_{j}\right) \neq\left(\frac{1}{2}, \frac{1}{2}\right)$, $j=1,2,3$, and let the conditions in (2.3) hold. Also, let $\phi, \phi_{0} \in$ $\Sigma_{s_{1}, \sigma_{2}, \sigma_{3}}^{\sigma_{1}, s_{2}, s_{3}}\left(\mathbf{R}^{3 d}\right) \backslash 0, \omega \in \mathscr{P}_{s_{1}, \sigma_{2}, \sigma_{3}}\left(\mathbf{R}^{3 d}\right)$, and let

$$
\omega_{R}(x, \xi, \eta, \zeta, y, z)=\omega(x, \xi, \eta) e^{-R\left(|\zeta|^{\frac{1}{\sigma_{1}}}+|y|^{\frac{1}{s_{2}}}+|z|^{\frac{1}{s_{3}}}\right)} .
$$

Then

$\Gamma_{(\omega)}^{\sigma_{1}, s_{2}, s_{3} ; 0}\left(\mathbf{R}^{3 d}\right)=\bigcap_{R>0}\left\{a \in\left(\sum_{s_{1}, \sigma_{2}, \sigma_{3}}^{\sigma_{1}, s_{2}, s_{3}}\right)^{\prime}\left(\mathbf{R}^{3 d}\right) ;\left\|\omega_{R}^{-1} V_{\phi} a\right\|_{L^{\infty, q}\left(\mathbf{R}^{3 d} \times \mathbf{R}^{3 d}\right)}<\infty\right\}$.

Proof. When $q=\infty$, (2.10) becomes

$$
\Gamma_{(\omega)}^{\sigma_{1}, s_{2}, s_{3} ; 0}=\bigcap_{R>0} M_{\left(1 / \omega_{R}\right)}^{\infty}\left(\mathbf{R}^{3 d}\right)
$$

which is a straightforward consequence of Proposition 2.7. Therefore it is enough to prove that

$$
\begin{aligned}
& \bigcap_{R>0} M_{\left(1 / \omega_{R}\right)}^{\infty}\left(\mathbf{R}^{3 d}\right) \\
& \quad=\bigcap_{R>0}\left\{a \in\left(\Sigma_{s_{1}, \sigma_{2}, \sigma_{3}}^{\sigma_{1}, s_{2}, s_{3}}\right)^{\prime}\left(\mathbf{R}^{3 d}\right) ;\left\|\omega_{R}^{-1} V_{\phi} a\right\|_{L^{\infty, q}\left(\mathbf{R}^{3 d} \times \mathbf{R}^{3 d}\right)}<\infty\right\} .
\end{aligned}
$$


Put

$$
\begin{aligned}
V_{0, a}(X, Y) & =\left|\left(V_{\phi_{0}} a\right)(x, \xi, \eta, \zeta, y, z)\right|, \quad V_{a}(X, Y)=\left|\left(V_{\phi} a\right)(x, \xi, \eta, \zeta, y, z)\right| \\
& \text { and } G(x, \xi, \eta, \zeta, y, z)=\left|\left(V_{\phi} \phi_{0}\right)(x, \xi, \eta, \zeta, y, z)\right|
\end{aligned}
$$

where $X=(x, \xi, \eta) \in \mathbf{R}^{3 d}$ and $Y=(\zeta, y, z) \in \mathbf{R}^{3 d}$. By Proposition 1.8 we have

$$
0 \leq G(x, \xi, \eta, \zeta, y, z) \lesssim e^{-R\left(|x|^{\frac{1}{s_{1}}}+|\xi|^{\frac{1}{\sigma_{2}}}+|\eta|^{\frac{1}{\sigma_{3}}}+|\zeta|^{\frac{1}{\sigma_{1}}}+|y|^{\frac{1}{s_{2}}}+|z|^{\frac{1}{s_{3}}}\right)}
$$

$x, \xi, \eta, \zeta, y, z \in \mathbf{R}^{d}$, for every $R>0$.

From [24, Lemma 11.3.3] (when extended to the duality between Gelfand-Shilov spaces and their dual spaces of distributions) it follows that $V_{a} \lesssim V_{0, a} * G$, so we obtain

$$
\left(\omega_{R}^{-1} \cdot V_{a}\right)(X, Y) \lesssim\left(\left(\omega_{c R}^{-1} \cdot V_{0, a}\right) * G_{1}\right)(X, Y),, \quad X, Y \in \mathbf{R}^{3 d}
$$

for some $G_{1}$ which satisfies (2.11), and for a constant $c>0$ independent of $R$. By applying the $L^{\infty}$-norm on the both sides of (2.12) we obtain

$$
\begin{aligned}
& \left\|\omega_{R}^{-1} V_{a}\right\|_{L^{\infty}\left(\mathbf{R}^{6 d}\right)}=\sup _{Y} \sup _{X}\left|\omega_{R}^{-1} V_{a}(X, Y)\right| \\
& \quad \lesssim \sup _{Y} \sup _{X}\left|\omega_{c R}^{-1} V_{0, a} * G_{1}(X, Y)\right| \\
& \lesssim \sup _{Y}\left(\iint\left(\sup _{X}\left(\omega_{c R}^{-1} \cdot V_{0, a}\right)\left(X, Y-Y_{1}\right)\right) G_{1}\left(X_{1}, Y_{1}\right) d X_{1} d Y_{1}\right) \\
& \quad \leq\left\|\omega_{c R}^{-1} \cdot V_{0, a}\right\|_{L^{\infty, q}}\left\|G_{1}\right\|_{L^{1, q^{\prime}}} \asymp\left\|\omega_{c R}^{-1} \cdot V_{0, a}\right\|_{L^{\infty, q}},
\end{aligned}
$$

wherefrom

$$
\begin{aligned}
\bigcap_{R>0}\left\{a \in\left(\Sigma_{s_{1}, \sigma_{2}, \sigma_{3}}^{\sigma_{1}, s_{2}, s_{3}}\right)^{\prime}\left(\mathbf{R}^{3 d}\right) ;\left\|\omega_{R}^{-1} V_{\phi} a\right\|_{L^{\infty}, q\left(\mathbf{R}^{3 d} \times \mathbf{R}^{3 d}\right)}\right. & <\infty\} \\
& \subset \bigcap_{R>0} M_{\left(1 / \omega_{R}\right)}^{\infty}\left(\mathbf{R}^{3 d}\right) .
\end{aligned}
$$


For the opposite inclusion we put $K_{j}=\omega_{j c R}^{-1} \cdot V_{0, a}, j=1,2$. By (2.12) and Minkowski's inequality we have

$$
\begin{aligned}
& \left\|\omega_{R}^{-1} \cdot V_{a}\right\|_{L^{\infty, q}}^{q} \lesssim \|\left(\left(K_{1} * G\right) \|_{L^{\infty, q}}^{q}\right. \\
& \lesssim \int \sup _{X}\left(\iint K_{1}\left(X-X_{1}, Y-Y_{1}\right) G\left(X_{1}, Y_{1}\right) d X_{1} d Y_{1}\right)^{q} d Y \\
& \quad \lesssim \int\left(\iint \sup \left(K_{2}\left(\cdot, Y-Y_{1}\right)\right) G\left(X_{1}, Y_{1}\right)\right. \\
& \left.\times e^{-c R\left(\left|\zeta-\zeta_{1}\right|^{\frac{1}{\sigma_{1}}}+\left|y-y_{1}\right|^{\frac{1}{s_{2}}}+\left|z-z_{1}\right|^{\frac{1}{s_{3}}}\right)} d X_{1} d Y_{1}\right)^{q} d Y \\
& \lesssim\left\|K_{2}\right\|_{L^{\infty}}^{q} \int\left(\iint G\left(X_{1}, Y_{1}\right) e^{-c R\left(\left|\zeta-\zeta_{1}\right|^{\frac{1}{\sigma_{1}}}+\left|y-y_{1}\right|^{\frac{1}{s_{2}}}+\left|z-z_{1}\right|^{\frac{1}{s_{3}}}\right)} d X_{1} d Y_{1}\right)^{q} d Y \\
& \lesssim\left\|K_{2}\right\|_{L^{\infty}}^{q} \equiv\left\|\omega_{2 c R}^{-1} \cdot V_{0, a}\right\|_{L^{\infty}}^{q} .
\end{aligned}
$$

Finally, by interchanging the roles of $\phi$ and $\phi_{0}$ we get

$$
\left\|\omega_{R}^{-1} \cdot V_{0, a}\right\|_{L^{\infty, q}} \lesssim\left\|\omega_{2 c R}^{-1} \cdot V_{a}\right\|_{L^{\infty}}
$$

i.e.

$$
\begin{aligned}
& \bigcap_{R>0} M_{\left(1 / \omega_{R}\right)}^{\infty}\left(\mathbf{R}^{3 d}\right) \\
& \quad \subset \bigcap_{R>0}\left\{a \in\left(\Sigma_{s_{1}, \sigma_{2}, \sigma_{3}}^{\sigma_{1}, s_{2}, s_{3}}\right)^{\prime}\left(\mathbf{R}^{3 d}\right) ;\left\|\omega_{R}^{-1} V_{\phi} a\right\|_{L^{\infty, q}\left(\mathbf{R}^{3 d} \times \mathbf{R}^{3 d}\right)}<\infty\right\},
\end{aligned}
$$

and the result follows.

We leave for the reader to write down Proposition 2.8 when the (Roumieu case) symbol class $\Gamma_{(\omega)}^{\sigma_{1}, s_{2}, s_{3}}\left(\mathbf{R}^{3 d}\right)$ is considered instead.

In [9, Theorem 4.1], it is shown that if $A$ is a $d \times d$-matrix with real entries, then the operator $e^{i\left\langle A D_{\xi}, D_{x}\right\rangle}$ is a homeomorphism between certain classes of symbols. We proceed with an analogous result in the context of the symbol class $\Gamma_{(\omega)}^{\sigma_{1}, s_{2}, s_{3} ; 0}\left(\mathbf{R}^{3 d}\right)$.

Theorem 2.9. Let $s_{j}, \sigma_{j}>0$ be such that the conditions in (2.3) hold, and let $r, t \in[0,1]$ be such that $r+t \leq 1$.

If $\omega \in \mathscr{P}_{s_{1}, \sigma_{2}, \sigma_{3}}^{0}\left(\mathbf{R}^{3 d}\right)$, then $a \in \Gamma_{(\omega)}^{\sigma_{1}, s_{2}, s_{3}}\left(\mathbf{R}^{3 d}\right)$ if and only if

$$
e^{-i\left\langle r D_{\xi}+t D_{\eta}, D_{x}\right\rangle} a \in \Gamma_{(\omega)}^{\sigma_{1}, s_{2}, s_{3}}\left(\mathbf{R}^{3 d}\right) .
$$

If $\omega \in \mathscr{P}_{s_{1}, \sigma_{2}, \sigma_{3}}\left(\mathbf{R}^{3 d}\right)$ instead, and if, in addition to (2.3),$\left(s_{j}, \sigma_{j}\right) \neq$ $\left(\frac{1}{2}, \frac{1}{2}\right), j=1,2,3$, then $a \in \Gamma_{(\omega)}^{\sigma_{1}, s_{2}, s_{3} ; 0}\left(\mathbf{R}^{3 d}\right)$ if and only if

$$
e^{-i\left\langle r D_{\xi}+t D_{\eta}, D_{x}\right\rangle} a \in \Gamma_{(\omega)}^{\sigma_{1}, s_{2}, s_{3} ; 0}\left(\mathbf{R}^{3 d}\right) .
$$


Proof. We give the proof for the Beurling case, and the Roumieu case is left for the reader.

We will use the result of Proposition 2.8. Therefore we fix a window function $\phi \in \Sigma_{s_{1}, \sigma_{2}, \sigma_{3}}^{\left.\sigma_{1}, s_{2}, \mathbf{R}^{3 d}\right)}$ and let $\phi_{r, t}=e^{-i\left\langle r D_{\xi}+t D_{\eta}, D_{x}\right\rangle} \phi$. Then, in view of Theorem $2.2(2), \phi_{r, t}$ belongs to $\Sigma_{s_{1}, \sigma_{2}, \sigma_{3}}^{\sigma_{1}, s_{2}, s_{3}}\left(\mathbf{R}^{3 d}\right)$.

By similar arguments as in the proof of Lemma 2.1, we get

$$
\begin{aligned}
\left|\left(V_{\phi_{r, t}}\left(e^{-i\left\langle r D_{\xi}+t D_{\eta}, D_{x}\right\rangle} a\right)\right)(x, \xi, \eta, \zeta, y, z)\right| \\
=\left|\left(V_{\phi} a\right)(x+r y+t z, \xi+r \zeta, \eta+t \zeta, \zeta, y, z)\right|,
\end{aligned}
$$

$x, \xi, \eta, \zeta, y, z \in \mathbf{R}^{d}$. Then using (2.13) and a change of variables argument, we get

$$
\left\|\omega_{0,0 ; R}^{-1} V_{\phi} a\right\|_{L^{p, q}}=\left\|\omega_{r, t ; R}^{-1} V_{\phi_{r, t}}\left(e^{-i\left\langle r D_{\xi}+t D_{\eta}, D_{x}\right\rangle} a\right)\right\|_{L^{p, q}},
$$

where

$\omega_{r, t ; R}(x, \xi, \eta, \zeta, y, z)=\omega(x+r y+t z, \xi+r \zeta, \eta+t \zeta) e^{-R\left(|y|^{\frac{1}{s_{2}}}+|z|^{\frac{1}{s_{3}}}+|\zeta|^{\frac{1}{\sigma_{1}}}\right)}$, and $p, q \in[1, \infty]$.

Hence Proposition 2.8, and the fact that there exists a constant $c>0$ such that

$$
\omega_{0,0 ; R+c} \lesssim \omega_{r, t ; R} \lesssim \omega_{0,0 ; R-c}
$$

give

$$
\begin{gathered}
a \in \Gamma_{(\omega)}^{\sigma_{1}, s_{2}, s_{3} ; 0}\left(\mathbf{R}^{3 d}\right) \quad \Leftrightarrow \quad\left\|\omega_{0,0 ; R}^{-1} V_{\phi} a\right\|_{L^{\infty}}<\infty \text { for every } R>0 \\
\Leftrightarrow \quad\left\|\omega_{r, t ; R}^{-1} V_{\phi_{r, t}}\left(e^{-i\left\langle r D_{\xi}+t D_{\eta}, D_{x}\right\rangle} a\right)\right\|_{L^{\infty}}<\infty \text { for every } R>0 \\
\Leftrightarrow\left\|\omega_{0,0 ; R}^{-1} V_{\phi_{r, t}}\left(e^{-i\left\langle r D_{\xi}+t D_{\eta}, D_{x}\right\rangle} a\right)\right\|_{L^{\infty}}<\infty \text { for every } R>0 \\
\Leftrightarrow \quad e^{-i\left\langle r D_{\xi}+t D_{\eta}, D_{x}\right\rangle} a \in \Gamma_{(\omega)}^{\sigma_{1}, s_{2}, s_{3} ; 0}\left(\mathbf{R}^{3 d}\right),
\end{gathered}
$$

and the result follows.

\section{CONTINUity OF BILINEAR PSEUdo-DifFERENTIAL OPERATORS WITH SYMBOLS OF GEVREY-REGULARITY AND INFINITE ORDER}

We first discuss the continuity of bilinear operators in $\operatorname{Op}\left(\Gamma_{(\omega)}^{\sigma_{1}, s_{2}, s_{3}}\right)$ and $\operatorname{Op}\left(\Gamma_{(\omega)}^{\sigma_{1}, s_{2}, s_{3} ; 0}\right)$ when acting on products modulation spaces. In particular, Theorem 3.1 can be considered as an extension of [46, Theorem $3.2]$ to bilinear operators and a more general class of weights.

Theorem 3.1. Let $s_{j}, \sigma_{j}>0$ be such that the conditions in (2.3) hold. Also, let $v_{1} \in \mathscr{P}_{s_{1}}^{0}\left(\mathbf{R}^{d}\right), v_{j} \in \mathscr{P}_{\sigma_{j}}^{0}\left(\mathbf{R}^{d}\right), j=2,3, \omega_{0}, \omega \in \mathscr{P}_{s_{1}, \sigma_{2}, \sigma_{3}}^{0}\left(\mathbf{R}^{3 d}\right)$, and let $\omega_{0}$ be $\otimes_{j=1}^{3} v_{j}$-moderate. Furthermore, let $r, t \in[0,1]$ such that $r+t \leq 1$, and let $p, q \in[1, \infty]$. If $a \in \Gamma_{\left(\omega_{0}\right)}^{\sigma_{1}, s_{2}, s_{3}}\left(\mathbf{R}^{3 d}\right)$, then there exists 
$R>0$ such that $\mathrm{Op}_{r, t}(a)$ is continuous from $M_{\left(\omega_{0} \omega\right)}^{p, q}\left(\mathbf{R}^{d}\right) \times M_{\left(1 / \omega_{R}\right)}^{\infty, \infty}\left(\mathbf{R}^{d}\right)$ to $M_{(\omega)}^{p, q}\left(\mathbf{R}^{d}\right)$, where

$$
\omega_{R}(x, \xi, \eta)=e^{-R\left(|x|^{\frac{1}{s_{1}}}+|\xi|^{\frac{1}{\sigma_{2}}}+|\eta|^{\frac{1}{\sigma_{3}}}\right)}, \quad x, \xi, \eta \in \mathbf{R}^{d} .
$$

Remark 3.2. We will use estimates similar to those obtained in the proof of [8, Theorem 6.1]. We observe that out arguments are anyway different since, in view of the fact that we employ Gevrey tpye symbols, we cannot rely on standard localization techniques. The idea is that for a fixed function $g$ in appropriate space of test functions, $a \in \Gamma_{(\omega)}^{\sigma_{1}, s_{2}, s_{3}}\left(\mathbf{R}^{3 d}\right)$, and $r=t=0$, the operator $\operatorname{Op}_{0,0}(a)(\cdot, g) \equiv T_{a}(\cdot, g)$ can be regarded as a linear pseudo-differential operator (with symbol depending on $g$ ), that is,

$$
\mathrm{Op}_{0,0}(a)(f, g)(x)=(2 \pi)^{-\frac{d}{2}} \int e^{i\langle x, \xi\rangle} a_{g}(x, \xi) \widehat{f}(\xi) d \xi
$$

where

$$
a_{g}(x, \xi)=(2 \pi)^{-\frac{d}{2}} \int e^{i\langle x, \eta\rangle} a(x, \xi, \eta) \widehat{g}(\eta) d \eta .
$$

If the symbol $a_{g}$ belongs to $\Gamma_{\left(\omega_{0}\right)}^{\sigma_{1}, s_{2}}\left(\mathbf{R}^{2 d}\right)$, then the continuity of $\mathrm{Op}_{0,0}(a)(\cdot, g)$ from $M_{\left(\omega_{0} \omega\right)}^{p . q}$ to $M_{(\omega)}^{p . q}$ follows by Proposition 1.10 .

Lemma 3.3. Let $s_{j}, \sigma_{j}>0$ be such that the conditions in (2.3) hold. Also, let $v_{1} \in \mathscr{P}_{s_{1}}^{0}\left(\mathbf{R}^{d}\right), v_{j} \in \mathscr{P}_{\sigma_{j}}^{0}\left(\mathbf{R}^{d}\right), j=2,3, \omega_{0}, \omega \in \mathscr{P}_{s_{1}, \sigma_{2}, \sigma_{3}}^{0}\left(\mathbf{R}^{3 d}\right)$, and let $\omega_{0}$ be $\otimes_{j=1}^{3} v_{j}$-moderate.

If $g \in \mathcal{S}_{s_{1}}^{\sigma_{1}}\left(\mathbf{R}^{d}\right)$ and $a \in \Gamma_{\left(\omega_{0}\right)}^{\sigma_{1}, s_{2}, s_{3}}\left(\mathbf{R}^{3 d}\right)$, then the symbol $a_{g}$ given by (3.1) belongs to $\Gamma_{(\omega)}^{\sigma_{1}, s_{2}}\left(\mathbf{R}^{2 d}\right)$, where $\omega(x, \xi) \equiv \omega_{0}(x, \xi, 0) \in \mathscr{P}_{s_{1}, \sigma_{2}}^{0}\left(\mathbf{R}^{2 d}\right)$.

Proof. By (3.1) it follows that $a_{g}$ is a smooth function. Indeed,

$$
(x, \xi, \eta) \mapsto e^{i\langle x, \eta\rangle} a(x, \xi, \eta) \widehat{g}(\eta)
$$

is a smooth mapping and

$$
\eta \mapsto \eta^{\gamma} e^{i\langle x, \eta\rangle} \partial_{x}^{\alpha} \partial_{\xi}^{\beta} a(x, \xi, \eta) \widehat{g}(\eta)
$$

is an integrable function for every $x, \xi, \alpha, \beta$ and $\gamma$.

Since $\widehat{g} \in \mathcal{S}_{\sigma_{1}}^{s_{1}}\left(\mathbf{R}^{d}\right)\left(\right.$ cf. Proposition 1.4) and since $\omega_{0} \in \mathscr{P}_{s_{1}, \sigma_{2}, \sigma_{3}}^{0}\left(\mathbf{R}^{3 d}\right)$, it follows that

$$
\begin{aligned}
& \left|\partial_{x}^{\alpha} \partial_{\xi}^{\beta} a_{g}(x, \xi)\right| \lesssim \sum_{\gamma \leq \alpha}\left(\begin{array}{c}
\alpha \\
\gamma
\end{array}\right) \int\left|\eta^{\gamma} \partial_{x}^{\alpha-\gamma} \partial_{\xi}^{\beta} a(x, \xi, \eta) \widehat{g}(\eta)\right| d \eta \\
& \lesssim \sum_{\gamma \leq \alpha}\left(\begin{array}{l}
\alpha \\
\gamma
\end{array}\right) \int h^{|\alpha+\beta-\gamma|}(\alpha-\gamma) !^{\sigma_{1}} \beta !^{s_{2}}\left|\omega_{0}(x, \xi, \eta) \eta^{\gamma} e^{-r|\eta|^{\frac{1}{\sigma_{1}}}}\right| d \eta \\
& \lesssim \sum_{\gamma \leq \alpha}\left(\begin{array}{l}
\alpha \\
\gamma
\end{array}\right) \int h^{|\alpha+\beta-\gamma|}(\alpha-\gamma) !^{\sigma_{1}} \beta !^{s_{2}} \omega_{0}(x, \xi, 0)\left|e^{r_{0}|\eta|^{\frac{1}{\sigma_{3}}}} \eta^{\gamma} e^{-r|\eta|^{\frac{1}{\sigma_{1}}}}\right| d \eta
\end{aligned}
$$


for every $r_{0}>0$, and some constants $r, h>0$. Since $r_{0}$ can be chosen such that $r_{0}<r$, and since

$$
\left|\eta^{\gamma} e^{-\left(r-r_{0}\right)|\eta|^{\frac{1}{\sigma_{1}}}}\right| \lesssim h^{|\gamma|} \eta^{!^{\sigma_{1}}} e^{-\frac{\left(r-r_{0}\right)}{2}|\eta|^{\frac{1}{\sigma_{1}}}}
$$

we get

$$
\begin{aligned}
& \left|\partial_{x}^{\alpha} \partial_{\xi}^{\beta} a_{g}(x, \xi)\right| \\
& \lesssim h^{|\alpha+\beta|} \beta !^{s_{2}} \sum_{\gamma \leq \alpha}\left(\begin{array}{l}
\alpha \\
\gamma
\end{array}\right)((\alpha-\gamma) ! \gamma !)^{\sigma_{1}} \int \omega_{0}(x, \xi, 0) e^{-\left(r-r_{0}\right)|\eta|^{\frac{1}{\sigma_{1}}}} d \eta, \\
& \lesssim(4 h)^{|\alpha+\beta|} \alpha !^{\sigma_{1}} \beta !^{s_{2}} \omega(x, \xi), \quad x, \xi \in \mathbf{R}^{d},
\end{aligned}
$$

for some constant $h>0$, where $\omega(x, \xi) \equiv \omega_{0}(x, \xi, 0) \in \mathscr{P}_{s_{1}, \sigma_{2}}^{0}\left(\mathbf{R}^{2 d}\right)$. This gives the desired result.

Proof of Theorem 3.1. In view of the invariance properties for the bilinear pseudo-differential operators given in Theorem 2.9, we may assume $r=t=0$ without loss of generality.

By Proposition 1.10 and Lemma 3.3, it follows that $\mathrm{Op}(a)(f, g)$ is a continuous mapping from $M_{\left(\omega_{0} \omega\right)}^{p . q}\left(\mathbf{R}^{d}\right) \times \mathcal{S}_{s_{1}}^{\sigma_{1}}\left(\mathbf{R}^{d}\right)$ to $M_{(\omega)}^{p . q}\left(\mathbf{R}^{d}\right)$. Now the result follows from Proposition 1.8 .

In a similar way, we get the result for the Beurling case. The details are left for the reader.

Theorem 3.4. Let $s_{j}, \sigma_{j}>0,\left(s_{j}, \sigma_{j}\right) \neq\left(\frac{1}{2}, \frac{1}{2}\right), j=1,2,3$, be such that the conditions in (2.3) hold. Also, let $v_{1} \in \mathscr{P}_{s_{1}}^{0}\left(\mathbf{R}^{d}\right), v_{j} \in \mathscr{P}_{\sigma_{j}}^{0}\left(\mathbf{R}^{d}\right)$, $j=2,3, \omega_{0}, \omega \in \mathscr{P}_{s_{1}, \sigma_{2}, \sigma_{3}}^{0}\left(\mathbf{R}^{3 d}\right)$, and let $\omega_{0}$ be $\otimes_{j=1}^{3} v_{j}$-moderate. Furthermore, let $r, t \in[0,1]$ such that $r+t \leq 1$, and let $p, q \in[1, \infty]$.

If $a \in \Gamma_{\left(\omega_{0}\right)}^{\sigma_{1}, s_{2}, s_{3} ; 0}\left(\mathbf{R}^{3 d}\right)$, then for any $R>0$ the operator $\mathrm{Op}_{r, t}(a)$ is continuous from $M_{\left(\omega_{0} \omega\right)}^{p, q}\left(\mathbf{R}^{d}\right) \times M_{\left(1 / \omega_{R}\right)}^{\infty, \infty}\left(\mathbf{R}^{d}\right)$ to $M_{(\omega)}^{p, q}\left(\mathbf{R}^{d}\right)$, where

$$
\omega_{R}(x, \xi, \eta)=e^{-R\left(|x|^{\frac{1}{s_{1}}}+|\xi|^{\frac{1}{\sigma_{2}}}+|\eta|^{\frac{1}{\sigma_{3}}}\right)}, \quad x, \xi, \eta \in \mathbf{R}^{d} .
$$

Finally, the characterization of Gelfand-Shilov spaces via modulation spaces gives the following result (cf. [26, 40, 47]), see also Proposition 1.8 .

Theorem 3.5. Let there be given $s, \sigma>0$ such that $s+\sigma \geq 1, v_{1} \in$ $\mathscr{P}_{s}^{0}\left(\mathbf{R}^{d}\right), v_{j} \in \mathscr{P}_{\sigma}^{0}\left(\mathbf{R}^{d}\right), j=2,3$, and $\omega_{0} \in \mathscr{P}_{s, \sigma, \sigma}^{0}\left(\mathbf{R}^{3 d}\right)$, such that $\omega_{0}$ is $\otimes_{j=1}^{3} v_{j}$-moderate. If $r, t \in[0,1]$, such that $r+t \leq 1$, and $a \in \Gamma_{(\omega)}^{\sigma, s, s}\left(\mathbf{R}^{3 d}\right)$ then $\mathrm{Op}_{r, t}(a)$ is continuous from $\mathcal{S}_{s}^{\sigma}\left(\mathbf{R}^{d}\right) \times \mathcal{S}_{s}^{\sigma}\left(\mathbf{R}^{d}\right)$ to $\mathcal{S}_{s}^{\sigma}\left(\mathbf{R}^{d}\right)$, and from $\left(\mathcal{S}_{s}^{\sigma}\right)^{\prime}\left(\mathbf{R}^{d}\right) \times\left(\mathcal{S}_{s}^{\sigma}\right)^{\prime}\left(\mathbf{R}^{d}\right)$ to $\left(\mathcal{S}_{s}^{\sigma}\right)^{\prime}\left(\mathbf{R}^{d}\right)$.

Proof. In view of Theorem 2.9, it is enough to consider the case when $r=t=0$, i.e. $\mathrm{Op}_{0,0}(a)$. 
By Proposition 1.11 and Remark [3.2, it is enough to show that $a_{g}$ given by (3.1) belongs to $\Gamma_{(\omega)}^{\sigma, s}\left(\mathbf{R}^{2 d}\right)$ for $\omega(x, \xi) \equiv \omega_{0}(x, \xi, 0) \in$ $\mathscr{P}_{s, \sigma}^{0}\left(\mathbf{R}^{2 d}\right)$. This follows from Lemma 3.3. Now the the continuity of $\mathrm{Op}_{r, t}(a)$ from $\mathcal{S}_{s}^{\sigma}\left(\mathbf{R}^{d}\right) \times \mathcal{S}_{s}^{\sigma}\left(\mathbf{R}^{d}\right)$ to $\mathcal{S}_{s}^{\sigma}\left(\mathbf{R}^{d}\right)$ follows from Theorem 3.1 and Proposition 1.8 .

The continuity of $\mathrm{Op}_{r, t}(a)$ from $\left(\mathcal{S}_{s}^{\sigma}\right)^{\prime}\left(\mathbf{R}^{d}\right) \times\left(\mathcal{S}_{s}^{\sigma}\right)^{\prime}\left(\mathbf{R}^{d}\right)$ to $\left(\mathcal{S}_{s}^{\sigma}\right)^{\prime}\left(\mathbf{R}^{d}\right)$ follows by duality.

The analogous result given below follows by similar arguments and is left for the reader.

Theorem 3.6. Let there be given $s, \sigma>0$ such that $s+\sigma \geq 1$ and $(s, \sigma) \neq\left(\frac{1}{2}, \frac{1}{2}\right)$. Also, let $v_{1} \in \mathscr{P}_{s}^{0}\left(\mathbf{R}^{d}\right), v_{j} \in \mathscr{P}_{\sigma}^{0}\left(\mathbf{R}^{d}\right), j=2,3$, and $\omega_{0} \in \mathscr{P}_{s, \sigma, \sigma}^{0}\left(\mathbf{R}^{3 d}\right)$, such that $\omega_{0}$ is $\otimes_{j=1}^{3} v_{j}$-moderate. If $r, t \in[0,1]$, such that $r+t \leq 1$, and $a \in \Gamma_{(\omega)}^{\sigma, s, s ; 0}\left(\mathbf{R}^{3 d}\right)$ then $\mathrm{Op}_{r, t}(a)$ is continuous from $\Sigma_{s}^{\sigma}\left(\mathbf{R}^{d}\right) \times \Sigma_{s}^{\sigma}\left(\mathbf{R}^{d}\right)$ to $\Sigma_{s}^{\sigma}\left(\mathbf{R}^{d}\right)$, and from $\left(\Sigma_{s}^{\sigma}\right)^{\prime}\left(\mathbf{R}^{d}\right) \times\left(\Sigma_{s}^{\sigma}\right)^{\prime}\left(\mathbf{R}^{d}\right)$ to $\left(\Sigma_{s}^{\sigma}\right)^{\prime}\left(\mathbf{R}^{d}\right)$.

\section{Appendix A. Proofs of some Auxiliary Results}

In this concluding section we collect the proofs of some technical results employed above.

Proof of Lemma 1.2. We give the proof for the case $\Sigma_{s}^{\sigma}\left(\mathbf{R}^{d}\right)$ (the Beurling case) only, and the (simpler) case $\mathcal{S}_{s}^{\sigma}\left(\mathbf{R}^{d}\right)$ (the Roumieu case) is left for the reader. For simplicity (to avoid the use of inequalities related to multi-indices) we here set $d=1$.

Note that

$$
\sup _{x}\left|x^{p} f^{(q)}(x)\right| \leq C k^{p} l^{q} p !^{s} q^{l^{\sigma}}, \quad \forall k, l>0,
$$

is equivalent to

$$
\sup _{x}\left|x^{p} f^{(q)}(x)\right| \leq C h^{p+q} p !^{s} q !^{\sigma}, \quad \forall h>0 .
$$

In fact, if the former condition holds, then we may put $l=k=h$ for any given $h>0$, so the later condition holds. If the later condition holds instead, then for any given $k, l>0$ we may choose $h=\min \{k, l\}$ to obtain

$$
\sup _{x}\left|x^{p} f^{(q)}(x)\right| \leq C \min \{k, l\}^{p+q} p !^{s} q !^{\sigma} \leq C k^{p} l^{q} p !^{s} q !^{\sigma},
$$

and the equivalence follows.

Assume that $f \in \Sigma_{s}^{\sigma}(\mathbf{R})$, that is, for every $p, q \in \mathbf{N}$,

$$
\sup _{x \in \mathbf{R}}\left|x^{p} f^{(q)}(x)\right| \lesssim k^{p} l^{q} p !^{s} q !^{\sigma}, \quad \forall k, l>0 .
$$

Put $F_{q}(x, l)=f^{(q)}(x) /\left(l^{q} q !^{\sigma}\right), x \in \mathbf{R}$. Then, by (A.1)

$$
\sup _{x} k^{-p} p !^{-s}|x|^{p}\left|F_{q}(x, l)\right|<\infty \Rightarrow \sup _{x} k^{-\frac{p}{s}} p !^{-1}|x|^{\frac{p}{s}}\left|F_{q}(x, l)\right|^{\frac{1}{s}} \leq C,
$$


for some constant $C>0$, uniformly in $p$, so that

$$
\sup _{x} \sum_{p \in \mathbb{N}}\left(\frac{\left(|x| k^{-1}\right)^{1 / s}}{2}\right)^{p} p !^{-1}\left|F_{q}(x, l)\right|^{1 / s} \leq C \sum_{p \in \mathbb{N}} \frac{1}{2^{p}} .
$$

For any given $h>0$ we may choose $k=(s / 2 h)^{s}$ to obtain

$$
\begin{aligned}
\sup _{x} \sum_{p \in \mathbb{N}}\left(\frac{\left(\left(|x|\left(\frac{2 h}{s}\right)^{s}\right)^{1 / s}\right)^{p}}{2^{p} p !}\left|F_{q}(x, l)\right|^{1 / s}\right. & \\
& =\sup _{x} \sum_{p \in \mathbb{N}} \frac{\left(|x|^{1 / s} \frac{h}{s}\right)^{p}}{p !}\left|F_{q}(x, l)\right|^{1 / s} \leq \tilde{C} .
\end{aligned}
$$

Therefore

$e^{\frac{h}{s}|x|^{1 / s}} \sup _{x}\left|F_{q}(x, l)\right|^{1 / s} \leq \tilde{C} \Rightarrow\left|F_{q}(x, l)\right|^{1 / s} \leq \tilde{C} e^{-\frac{h}{s}|x|^{1 / s}}, \quad \forall h, l>0$,

and we conclude that

$$
\frac{\left|f^{(q)}(x)\right|}{l^{q} q !^{\sigma}} \leq \tilde{C}^{s} e^{-h|x|^{1 / s}} \Leftrightarrow\left|f^{(q)}(x)\right| \leq C l^{q} q !^{\sigma} e^{-h|x|^{1 / s}}, \quad \forall h, l>0,
$$

which proves the first part of Lemma.

Now, assume that (1.4) holds, so that for $F_{q}(x, l)=f^{(q)}(x) /\left(l^{q} q !^{\sigma}\right)$ we have

$$
\left|F_{q}(x, l)\right|^{1 / s} e^{\frac{h}{s}|x|^{1 / s}} \leq C, \quad \forall h, l>0,
$$

for some $C>0$ uniformly in $x \in \mathbf{R}$. Therefore

$$
\sup _{x} \sum_{p \in \mathbb{N}} \frac{1}{p !}\left(\frac{h}{s}\right)^{p}|x|^{p / s}\left|F_{q}(x, l)\right|^{1 / s}<\infty
$$

which implies

$$
\frac{1}{p !}\left(\frac{h}{s}\right)^{p}|x|^{p / s}\left|F_{q}(x, l)\right|^{1 / s}<\infty \Leftrightarrow \frac{1}{p ! s}\left(\frac{h}{s}\right)^{p s}|x|^{p}\left|F_{q}(x, l)\right|<\infty,
$$

that is

$$
\left|x^{p} f^{(q)}(x)\right| \leq C\left(\frac{s^{s}}{h^{s}}\right)^{p} l q p !^{s} q !^{\sigma}, \quad \forall h, l>0 .
$$

Finally, for a given $k>0$ by choosing $h=s / k^{1 / s}$, we have $k=(s / h)^{s}$ and

$$
\left|x^{p} f^{(q)}(x)\right| \leq C k^{p} l^{q} p !^{s} q !^{\sigma},
$$

which completes the proof.

Proof of Proposition 1.7. It is enough to prove (2) for every $0<s_{j}<1$, $j=1, \ldots, k$. In addition, we assume that $\left(s_{0}, \ldots, s_{k}\right) \neq(1 / 2, \ldots, 1 / 2)$, and leave the necessary modifications when $\left(s_{0}, \ldots, s_{k}\right)=(1 / 2, \ldots, 1 / 2)$ for the reader. 
Let $\phi_{0} \in \Sigma_{1-s_{0}, \ldots, 1-s_{k}}^{s_{0}, \ldots, s_{k}}\left(\mathbf{R}^{d_{0}+\cdots+d_{k}}\right) \backslash 0$, and $\phi=\left|\phi_{0}\right|^{2}$. Then $\phi \in_{1-s_{0}, \ldots, 1-s_{k}}^{s_{0}, s_{k}}$ $\left(\mathbf{R}^{d_{0}+\cdots+d_{k}}\right) \backslash 0$, i.e.

$$
\begin{array}{r}
\left|\partial_{x}^{\alpha_{0}} \partial_{\xi_{1}}^{\alpha_{1}} \ldots \partial_{\xi_{k}}^{\alpha_{k}} \phi\left(x, \xi_{1}, \ldots, \xi_{k}\right)\right| \lesssim h^{\left|\alpha_{0}+\alpha_{1}+\cdots+\alpha_{k}\right|} \prod_{j=0}^{k} \alpha_{j} !^{s_{j}} \\
\quad \times e^{-c\left(|x|^{\frac{1}{1-s_{0}}}+\left|\xi_{1}\right|^{\frac{1}{1-s_{1}}+\cdots+\left|\xi_{k}\right|^{\frac{1}{1-s_{k}}}}\right.},
\end{array}
$$

for every $h>0$ and $c>0$.

Now let $\omega_{0}=\omega * \phi$. Then we have

$$
\begin{aligned}
& \left|\partial_{x}^{\alpha_{0}} \partial_{\xi_{1}}^{\alpha_{1}} \ldots \partial_{\xi_{k}}^{\alpha_{k}} \omega_{0}\left(x, \xi_{1}, \ldots, \xi_{k}\right)\right| \\
& =\mid \int_{\mathbf{R}^{d_{0}+\cdots+d_{k}}} \omega\left(x-y, \xi_{1}-\eta_{1}, \ldots, \xi_{k}-\eta_{k}\right) \\
& \times\left(\partial_{x}^{\alpha_{0}} \partial_{\xi_{1}}^{\alpha_{1}} \ldots \partial_{\xi_{k}}^{\alpha_{k}} \phi\right)\left(y, \eta_{1}, \ldots, \eta_{k}\right) d y d \eta_{1} \ldots d \eta_{k} \mid \\
& \lesssim h^{\left|\alpha_{0}+\alpha_{1}+\cdots+\alpha_{k}\right|} \prod_{j=0}^{k} \alpha_{j} !^{s_{j}} \times \iint \cdots \int \omega\left(x-y, \xi_{1}-\eta_{1}, \ldots, \xi_{k}-\eta_{k}\right) \\
& \times e^{-c\left(|y|^{\frac{1}{1-s_{0}}}+\left|\eta_{1}\right|^{\frac{1}{1-s_{1}}}+\cdots+\left|\eta_{k}\right|^{\left.\frac{1}{1-s_{k}}\right)}\right.} d y d \eta_{1} \cdots d \eta_{k} \\
& \lesssim h^{\left|\alpha_{0}+\alpha_{1}+\cdots+\alpha_{k}\right|} \prod_{j=0}^{k} \alpha_{j} !^{s_{j}} \times \omega\left(x, \xi_{1}, \ldots, \xi_{k}\right) \\
& \times \iint \cdots \int e^{-\frac{c}{2}\left(|y|^{\frac{1}{1-s_{0}}}+\left|\eta_{1}\right|^{\frac{1}{1-s_{1}}}+\cdots+\left|\eta_{k}\right|^{\frac{1}{1-s_{k}}}\right)} d y d \eta_{1} \ldots d \eta_{k} \\
& \asymp h^{\left|\alpha_{0}+\alpha_{1}+\cdots+\alpha_{k}\right|} \prod_{j=0}^{k} \alpha_{j} !^{s_{j}} \times \omega\left(x, \xi_{1}, \ldots, \xi_{k}\right),
\end{aligned}
$$

where we used (1.8), (1.9), and the fact that for any $r, c>0$ and $s \in(0,1)$ it holds $e^{r|\cdot|} \lesssim e^{\frac{c}{2}|\cdot|^{1 / s}}$. This gives the first part of $(2)$ and $\omega_{0} \lesssim \omega$, by choosing $\alpha_{j}=0, j=0, \ldots, k$.

Now, for $\omega_{0} \gtrsim \omega$ we note that (1.8) and (1.9) imply

$$
\begin{aligned}
\omega\left(x-y, \xi_{1}-\eta_{1}, \ldots, \xi_{k}-\eta_{k}\right) \gtrsim & \frac{\omega\left(x, \xi_{1}, \ldots, \xi_{k}\right)}{v\left(-y,-\eta_{1}, \ldots,-\eta_{k}\right)} \\
& \gtrsim \omega\left(x, \xi_{1}, \ldots, \xi_{k}\right) e^{-r\left(|y|+\left|\eta_{1}\right|+\cdots+\left|\eta_{k}\right|\right)},
\end{aligned}
$$


for some $r>0$. Therefore,

$$
\begin{aligned}
& \left|\omega_{0}\left(x, \xi_{1}, \ldots, \xi_{k}\right)\right|=\left|\omega * \phi\left(x, \xi_{1}, \ldots, \xi_{k}\right)\right| \\
& \begin{aligned}
\left|\iint \cdots \int \omega\left(x-y, \xi_{1}-\eta_{1}, \ldots, \xi_{k}-\eta_{k}\right) \phi\left(y, \eta_{1}, \ldots, \eta_{k}\right) d y d \eta_{1} \cdots d \eta_{k}\right| \\
\gtrsim \omega\left(x, \xi_{1}, \ldots, \xi_{k}\right) \\
\times \iint \cdots \int e^{-r\left(|y|^{\frac{1}{1-s_{0}}}+\left|\eta_{1}\right|^{\frac{1}{1-s_{1}}}+\cdots+\left|\eta_{k}\right|^{\frac{1}{1-s_{k}}}\right)} \phi\left(y, \eta_{1}, \ldots, \eta_{k}\right) d y d \eta_{1} \cdots d \eta_{k} \\
\asymp \omega\left(x, \xi_{1}, \ldots, \xi_{k}\right),
\end{aligned}
\end{aligned}
$$

for some $r>0$, so that $\omega_{0} \gtrsim \omega$, and (1) follows. This also gives the second part of $(2)$.

Proof of Proposition 2.4. It is no restriction to assume that $r_{2}=t_{2}=$ 0 . Let $(r, t)=\left(r_{1}, t_{1}\right)$. The proof is a straightforward application of the Fourier inversion formula, see also [48, Proposition 1.1] and [27, Section $18.5]$.

The equality $\mathrm{Op}_{r, t}(a)=\mathrm{Op}(b)$ is the same as

$$
\begin{gathered}
\mathscr{F}_{2,3}(b(x, \cdot, \cdot \cdot))(y-x, z-x)=\mathscr{F}_{2,3}(a(x+r(y-x)+t(z-x), \cdot, \cdot \cdot))(y-x, z-x) \\
\Leftrightarrow \\
\iint b\left(x, \xi_{1}, \eta_{1}\right) e^{i\left(\left\langle y, \xi_{1}\right\rangle+\left\langle z, \eta_{1}\right\rangle\right)} d \xi_{1} d \eta_{1} \\
=\iint a\left(x+r y+t z, \xi_{1}, \eta_{1}\right) e^{i\left(\left\langle y, \xi_{1}\right\rangle+\left\langle z, \eta_{1}\right\rangle\right)} d \xi_{1} d \eta_{1} \\
\Leftrightarrow \\
b(x, \xi, \eta)=\frac{1}{(2 \pi)^{2 d}} \iiint \int a\left(x+r y+t z, \xi_{1}, \eta_{1}\right) e^{i\left(\left\langle y, \xi_{1}-\xi\right\rangle+\left\langle z, \eta_{1}-\eta\right\rangle\right)} d y d z d \xi_{1} d \eta_{1} .
\end{gathered}
$$

Let $Y=(y, z), Y_{2}=\left(y_{2}, z_{2}\right), \Xi=(\xi, \eta)$ and $\Xi_{1}=\left(\xi_{1}, \eta_{1}\right)$. Then by the Fourier inversion formula we get

$$
\begin{gathered}
b(x, \xi, \eta)= \\
(2 \pi)^{-\frac{7 d}{2}} \iiint \iint \widehat{a}\left(\zeta, y_{2}, z_{2}\right) e^{i\left(\langle x+r y+t z, \zeta\rangle+\left\langle Y_{2}, \Xi_{1}\right\rangle+\left\langle Y, \Xi_{1}-\Xi\right\rangle\right)} d z_{2} d y_{2} d \zeta d Y d \Xi_{1} \\
=(2 \pi)^{-\frac{3 d}{2}} \iiint \widehat{a}\left(\zeta, y_{2}, z_{2}\right) e^{i\left(\langle x, \zeta\rangle-\left\langle r y_{2}+t z_{2}, \zeta\right\rangle+\left\langle y_{2}, \xi\right\rangle+\left\langle z_{2}, \eta\right\rangle\right)} d z_{2} d y_{2} d \zeta \\
=(2 \pi)^{-\frac{3 d}{2}} \iiint e^{i\left(\left\langle y_{2}, \xi\right\rangle+\left\langle z_{2}, \eta\right\rangle+\langle x, \zeta\rangle\right)}\left(e^{-i\left\langle r y_{2}+t z_{2}, \zeta\right\rangle} \widehat{a}\left(\zeta, y_{2}, z_{2}\right)\right) d z_{2} d y_{2} d \zeta \\
=e^{-i\left\langle r D_{\xi}+t D_{\eta}, D_{x}\right\rangle} a(x, \xi, \eta),
\end{gathered}
$$

which gives the result. 


\section{ACKNOWLEDGEMENTS}

The authors are grateful to professor Joachim Toft for valuable remarks and suggestions. N. Teofanov is partially supported by MPNTR Project No 174024.

\section{REFERENCES}

[1] A. Abdeljawad, M. Cappiello, J. Toft, Pseudo-differential calculus in an anisotropic Gelfand-Shilov setting, Integr. Equ. Oper. Theory (2019) 91: 26.

[2] A. Abdeljawad, S. Coriasco, J. Toft, Liftings for ultra-modulation spaces, and one-parameter groups of Gevrey type pseudo-differential operators, (preprint), arXiv:1712.04338.

[3] A. Abdeljawad, J. Toft, Anisotropic Gevrey-Hörmander pseudo-differential operators on modulation spaces, (preprint), arXiv:1806.10002

[4] Á. Bényi, T. Oh, On a Class of Bilinear Pseudodifferential Operators, Journal of Function Spaces and Applications, vol. 2013, Article ID 560976, 5 pages, 2013. https://doi.org/10.1155/2013/560976.

[5] Á. Bényi, K. A. Okoudjou, Bilinear pseudodifferential operators on modulation spaces, J. Fourier Anal. Appl. 10 (2004), 301-313.

[6] Á. Bényi, K. A. Okoudjou, Modulation spaces estimates for multilinear pseudodifferential operators, Studia Math., 172 (2006), 169-180.

[7] Á. Bényi, K. Groechenig, C. Heil, K. Okoudjou, Modulation spaces and a class of bounded multilinear pseudodifferential operators, J. Operator Theory, 54 (2005), 389-401.

[8] Á. Bényi, D. Maldonado, V. Naibo, R.H. Torres, On the Hörmander classes of bilinear pseudodifferential operators, Integral Equations and Operator Theory 67 (2010), 341-364.

[9] M. Cappiello, J. Toft, Pseudo-differential operators in a Gelfand-Shilov setting, Math. Nachr. 290 (2017), 738-755.

[10] J. Chung, S.-Y. Chung, D. Kim, Characterizations of the Gelfand-Shilov spaces via Fourier transforms, Proc. Amer. Math. Soc. 124 (1996), 21012108.

[11] R.R. Coifman, Y. Meyer, Au delá des opérateurs pseudo-differentiels, Astérisque 57, Société Math. de France (1978)

[12] E. Cordero, S. Pilipović, L. Rodino, N. Teofanov, Localization operators and exponential weights for modulation spaces. Mediterr. J. Math. 2 (2005), 381394.

[13] E. Cordero, K. A. Okoudjou, Multilinear localization operators, J. Math. Anal. Appl. 325 (2007), 1103-1116.

[14] H. G. Feichtinger, Banach spaces of distributions of Wiener's type and interpolation, in: P. Butzer, B. Sz. Nagy and E. Görlich (Eds), Proc. Conf. Oberwolfach, Functional Analysis and Approximation, August 1980, Int. Ser. Num. Math. 69 Birkhäuser Verlag, Basel, Boston, Stuttgart, 1981, pp. 153-165. 
[15] H. G. Feichtinger, Banach convolution algebras of Wiener's type, in: Proc. Functions, Series, Operators in Budapest, Colloquia Math. Soc. J. Bolyai, North Holland Publ. Co., Amsterdam Oxford NewYork, 1980.

[16] H. G. Feichtinger, Modulation spaces on locally compact abelian groups. Technical report, University of Vienna, Vienna, 1983; also in: M. Krishna, R. Radha, S. Thangavelu (Eds) Wavelets and their applications, Allied Publishers Private Limited, NewDehli Mumbai Kolkata Chennai Hagpur Ahmedabad Bangalore Hyderbad Lucknow, 2003, pp.99-140.

[17] H. G. Feichtinger, K. H. Gröchenig, Banach spaces related to integrable group representations and their atomic decompositions, I, J. Funct. Anal. 86 (1989), 307-340.

[18] H. G. Feichtinger, K. H. Gröchenig, Banach spaces related to integrable group representations and their atomic decompositions, II, Monatsh. Math. 108 (1989), 129-148.

[19] H. G. Feichtinger, K. H. Gröchenig, Gabor frames and time-frequency analysis of distributions, J. Functional Anal. (2) 146 (1997), 464-495.

[20] Y. V. Galperin, S. Samarah, Time-frequency analysis on modulation spaces $M_{m}^{p, q}, 0<p, q \leq \infty$, Appl. Comput. Harmon. Anal. 16 (2004), 1-18.

[21] I. M. Gelfand, G. E. Shilov, Generalized functions, I-III, Academic Press, NewYork London, 1968.

[22] L. Grafakos, R.H. Torres, Multilinear Calderón-Zygmund theory, Adv. Math., 165 (2002), 124-164/

[23] T. Gramchev, Gelfand-Shilov spaces: structural properties and applications to pseudodifferential operators in $\mathbb{R}^{n}$, in Quantization, PDEs, and geometry, 1-68, Oper. Theory Adv. Appl. 251, Birkhäuser/Springer, 2016.

[24] K. H. Gröchenig, Foundations of Time-Frequency Analysis, Birkhäuser, Boston, 2001.

[25] K. Gröchenig, Weight functions in time-frequency analysis in: L. Rodino, M. W. Wong (Eds) Pseudodifferential Operators: Partial Differential Equations and Time-Frequency Analysis, Fields Institute Comm., 52 2007, 343-366.

[26] K. Gröchenig, G. Zimmermann, Spaces of test functions via the STFT J. Funct. Spaces Appl. 2 (2004), 25-53.

[27] L. Hörmander, The Analysis of Linear Partial Differential Operators, vol I,II, III, Springer-Verlag, Berlin Heidelberg, 1983, 1985.

[28] K. Koezuka, N. Tomita, Bilinear Pseudo-differential Operators with Symbols in $B S_{1,1}^{m}$ on Triebel-Lizorkin Spaces, J. Fourier Anal. Appl, 24 2018, 309 319 .

[29] Z. Lozanov-Crvenković, D. Perišić, M. Tasković, Gelfand-Shilov spaces, Structural and Kernel theorems, Preprint. arXiv:0706.2268v2

[30] F. Nicola, L. Rodino, Global Pseudo-differential calculus on Euclidean spaces, Pseudo-Differential Operators. Theory and Applications 4, Birkhäuser Verlag (2010)

[31] S. Molahajloo, K. A. Okoudjou, G. E. Pfander, Boundedness of Multilinear Pseudodifferential Operators on Modulation Spaces, J. Fourier Anal. Appl., 22 (2016), 1381-1415. 
[32] S. Pilipović, Tempered Ultradistributions, Bollettino U.M.I. (7) 2-B (1988), 235-251.

[33] C. Pfeuffer, J. Toft, Compactness Properties for Modulation Spaces, J. Complex Anal. Oper. Theory (2019), https://doi.org/10.1007/s11785-019-009034

[34] B. Prangoski, Pseudodifferential operators of infinite order in spaces of tempered ultradistributions, Journal of Pseudo-Differential Operators and Applications, 4 (4), 495-549 (2013)

[35] M. Ruzhansky, M. Sugimoto, J. Toft, N. Tomita, Changes of variables in modulation and Wiener amalgam spaces, Math. Nachr. 284, (2011) 20782092.

[36] N. Teofanov, Ultradistributions and time-frequency analysis, in: P. Boggiatto, L. Rodino, J. Toft, M.W. Wong, (Eds) Oper. Theory Adv. Appl., 164, 173-191, Birkhäuser, Verlag (2006)

[37] N. Teofanov, Modulation spaces, Gelfand-Shilov spaces and pseudodifferential operators, Sampl. Theory Signal Image Process, 5 (2006), 225-242.

[38] N. Teofanov, Gelfand-Shilov spaces and localization operators, Funct. Anal. Approx. Comput. 2 (2015), 135-158.

[39] N. Teofanov, Bilinear localization operators on modulation spaces, J. Funct. Spaces 2018, Art. ID 7560870, 10 pp.

[40] N. Teofanov, The Grossmann-Royer transform, Gelfand-Shilov spaces, and continuity properties of localization operators on modulation spaces, in: L. Rodino, J. Toft (eds), Mathematical Analysis and Applications - Plenary Lectures, Springer Nature, 2018, pp. 161-207,

[41] N. Teofanov, Continuity properties of multilinear localization operators on modulation spaces, in P. Boggiatto, E. Cordero, H. Feichtinger, M. de Gosson, F. Nicola, A. Oliaro, A. Tabacco (eds), Landscapes of Time-frequency Analysis, Springer Nature (2019)

[42] J. Toft, Subalgebras to a Wiener type Algebra of Pseudo-Differential operators, Ann. Inst. Fourier (5) 51 (2001), 1347-1383.

[43] J. Toft, Continuity properties for modulation spaces with applications to pseudo-differential calculus, I, J. Funct. Anal. (2), 207 (2004), 399-429.

[44] J. Toft, Continuity properties for modulation spaces with applications to pseudo-differential calculus, II, Ann. Global Anal. Geom., 26 (2004), 73106.

[45] J. Toft, Continuity and Schatten properties for pseudo-differential operators on modulation spaces in: J. Toft, M. W. Wong, H. Zhu (Eds) Modern Trends in Pseudo-Differential Operators, Operator Theory: Advances and Applications 172, Birkhäuser Verlag, Basel, 2007, pp. 173-206.

[46] J. Toft, Pseudo-differential operators with smooth symbols on modulation spaces, Cubo, 11 (2009), 87-107.

[47] J. Toft, The Bargmann transform on modulation and Gelfand-Shilov spaces, with applications to Toeplitz and pseudo-differential operators, J. PseudoDiffer. Oper. Appl. 3 (2012), 145-227.

[48] J. Toft, Matrix parameterized pseudo-differential calculi on modulation spaces in: M. Oberguggenberger, J. Toft, J. Vindas, P. Wahlberg (eds), 
Generalized functions and Fourier analysis, Operator Theory: Advances and Applications Vol 260, Birkhäuser/Springer, Basel, 2017, pp. 215-235.

[49] J.Toft, Continuity of Gevrey-Hörmander pseudo-differential operators on modulation spaces, J. Pseudo-Differ. Oper. Appl. (2019) https://doi.org/10.1007/s11868-018-0273-9

[50] G. Tranquilli, Global normal forms and global properties in function spaces for second order Shubin type operators PhD Thesis, 2013.

Department of Mathematics, University of Turin, Italy

E-mail address: ahmed.abdeljawad@unito.it

Department of Mathematics, University of Turin, Italy

E-mail address: sandro.coriasco@unito.it

Department of Mathematics and Informatics, University of Novi SAD, SERBIA

E-mail address: nenad.teof anov@dmi.uns.ac.rs 